\title{
Two-Server Password-Only Authenticated Key Exchange
}

\author{
Jonathan Katz* $\quad$ Philip MacKenzie ${ }^{\dagger} \quad$ Gelareh Taban ${ }^{\ddagger} \quad$ Virgil Gligor $^{\S}$
}

\begin{abstract}
Typical protocols for password-based authentication assume a single server that stores all the information (e.g., the password) necessary to authenticate a user. An inherent limitation of this approach, assuming low-entropy passwords are used, is that the user's password is exposed if this server is ever compromised. To address this issue, it has been suggested to share a user's password information among multiple servers, and to have these servers cooperate (possibly in a threshold manner) when the user wants to authenticate.

We show here a two-server version of the password-only key-exchange protocol of Katz, Ostrovsky, and Yung (the KOY protocol). Our work gives the first secure two-server protocol for the password-only setting (in which the user need remember only a password, and not the servers' public keys), and is the first two-server protocol (in any setting) with a proof of security in the standard model. Our work thus fills a gap left by the work of MacKenzie et al. (J. Crypto 2006) and Di Raimondo and Gennaro (JCSS 2006). As an additional benefit of our work, we show modifications that improve the efficiency of the original KOY protocol.
\end{abstract}

\section{Introduction}

Humans typically choose "weak", low-entropy passwords, while standard authentication protocols assume the use of cryptographic (i.e., high-entropy) secrets. Unfortunately, protocols designed and proven secure in the latter setting are generally insecure in the former context because these protocols are not resistant to off-line dictionary attacks in which an eavesdropping adversary derives information about the password from observed transcripts of login sessions. In recent years, much attention has focused on designing password-based authenticated key-exchange protocols resistant to such attacks. (We remark that on-line dictionary attacks - in which an adversary simply attempts to login repeatedly, trying each possible password - cannot be prevented by cryptographic means but can be dealt with using other methods outside the scope of this work.)

Means of protecting against off-line dictionary attacks in a single-server setting were first suggested by Gong et al. [21] in a "hybrid", PKI-based model where users are required to store the server's public key in addition to a password. Bellovin and Merritt [5] were the first to suggest protocols for password-only authenticated key exchange (PAKE), where users are required to store only a short password. These initial works (and others $[6,23,28,34]$ ) were relatively informal and did not provide definitions or proofs of security. Subsequently, formal definitions and provably

\footnotetext{
*Dept. of Computer Science, University of Maryland. Work supported by NSF grants \#0447075 and \#0310751.

${ }^{\dagger}$ Google labs. Work done while at DoCoMo USA Labs.

${ }^{\ddagger}$ Work done while at the University of Maryland.

${ }^{\S}$ Dept. of Electrical and Computer Engineering, Carnegie Mellon University. Work done while at the University of Maryland.
} 
secure protocols for the "hybrid" model were given $[22,7]$, followed by models for the password-only setting $[1,8,20]$ and associated protocols with proofs of security in the random oracle/ideal cipher models $^{1}[1,8,30]$ or in the standard model $[26,20,19,25]$. (The protocols of $[26,19,25]$ assume some public information which is available to all parties. Since this information can be hard-coded into implementations of the protocol, clients do not need to memorize or store any high-entropy, cryptographic information as they are required to do in the PKI-based setting.)

Although the above protocols protect against off-line dictionary attacks, they do nothing to mitigate the concern that an adversary might obtain users' passwords via server compromise. Such attacks represent a serious threat since they are potentially cost-effective (in that an adversary might be able to obtain thousands of users' passwords by corrupting a single, poorly-protected server), and because users frequently utilize the same password at multiple sites. It is easy to show that it is impossible to protect against server compromise when a single server holds the information needed to authenticate a user (assuming the only secret information held by the user is a low-entropy password). To protect against server compromise, Ford and Kaliski [18] thus proposed using a threshold protocol in which the authentication functionality is distributed across $n$ servers who cooperate to authenticate a user, and who obtain independent session keys (shared with the user) following a successful authentication. Their protocol, which is designed in the PKI-based model, remains secure (and, in particular, an adversary learns nothing about users' passwords other than what it learns from its on-line password guesses) as long as $n-1$ or fewer servers are compromised. Jablon [24] gave a protocol with similar functionality in the password-only setting. Neither of these works, however, include rigorous definitions or proofs of security.

Subsequent to the work of Ford-Kaliski and Jablon, a number of provably secure protocols for threshold password-based authentication have been given. We summarize what is known:

- MacKenzie et al. [31] showed a protocol in the "hybrid", PKI-based setting which requires only $t$ out of $n$ servers to cooperate in order to authenticate a user, for any values of $t, n$ (of course, security is only obtained as long as $t-1$ or fewer servers are compromised). They prove security for their protocol in the random oracle model.

- Di Raimondo and Gennaro [16] proposed a protocol in the password-only setting with a proof of security in the standard model. (A second protocol given in their paper, which we will not discuss further, achieves the weaker functionality in which the user shares the same session key with all the servers.) Their protocol requires fewer than $1 / 3$ of the servers to be compromised (i.e., they require $t<n / 3$ ) and thus does not give a solution for the two-server case. $^{2}$ (In general, threshold cryptosystems for the two-party case do not follow immediately from threshold solutions that require honest majority.)

- Brainard et al. [9] developed a two-server protocol, a variant of which has been proven secure in the random oracle model [33]. These protocols assume the PKI-based setting since they require a "secure channel" between the client and the server(s) which must presumably be implemented using public-key techniques.

\footnotetext{
${ }^{1}$ In the random oracle model [2], parties are assumed to have "black-box" access to a random function; the ideal cipher model assumes that parties have "black-box" access to a random keyed permutation. In practice, a random oracle is instantiated by a cryptographic hash function. It is known [10], however, that protocols secure in the random oracle model may be insecure for any choice of hash function.

${ }^{2}$ The approach in their paper does not extend to the case $t \geq n / 3$. The authors mention (without details) that "[i]t is possible to improve the fault-tolerance to $t<n / 2 \ldots$ ", but even this would not imply a two-server solution.
} 


\subsection{Our Contributions}

We show here a two-server protocol for password-only authenticated key exchange, with a proof of security in the standard model. Ours is the first provably secure two-server protocol in the password-only setting, and is the first two-server protocol (in any setting) with a proof of security in the standard model. Our protocol extends and builds upon the (single-server) password-based key-exchange protocol of Katz, Ostrovsky, and Yung [26] (the KOY protocol). As an additional benefit of our work, we show two modifications which improve the efficiency of the original KOY protocol even in the single-server case. (One of these modifications was also used in [11].)

In Section 4 we describe a "basic" two-server protocol which is secure against a passive (i.e., "honest-but-curious") adversary who has access to the entire state of one of the servers throughout its execution of the protocol, but cannot cause this server to deviate from its prescribed behavior. (Even in the this case, however, the adversary is assumed to control all communication between the client and the servers.) This protocol is interesting in its own right (when the assumption on adversarial behavior is warranted), and serves as a useful prelude to our second result. In Section 5 we show how to modify the basic protocol so as to achieve security against an active adversary who may cause a corrupted server to deviate arbitrarily from the protocol.

The protocols we construct are relatively efficient. Each party in the basic two-server protocol performs (roughly) twice the amount of work as in the original KOY protocol. For the protocol secure against active adversaries, the work of the client stays the same but the work of the servers increases by a factor of approximately 6. (More explicit calculations of the computational cost are given in the appropriate sections.) This does not take into account potential efficiency improvements such as off-line computation or pre-processing.

\section{Definitions and Preliminaries}

We assume the reader is familiar with the model of Bellare et al. [1] (building on [3, 4]) for passwordbased key exchange in the single-server case. Here, we generalize their model and present formal definitions for two-server protocols. While the model presented here is largely equivalent to the model proposed by MacKenzie et al. [31] (with the main difference being that we do not assume a PKI), we can simplify matters a bit since we focus on the two-server setting exclusively. For convenience we first describe the model for the case of a "passive" adversary corrupting one of the servers, and then discuss briefly the modifications needed to handle an "active" adversary. (As discussed below, in both the "passive" and "active" cases the adversary is free to interfere with all communication between the client and the servers. These cases only differ in the power of the adversary to control the actions of the corrupted servers: specifically, a "passive" adversary is unable to control the actions of corrupted servers, whereas an "active" adversary can.)

We first present a general overview of the system. For simplicity, we assume that every client $C$ in the system shares its password $p w$ with exactly two servers $A$ and $B$. In this case we say that servers $A$ and $B$ are associated with $C$. (A single server may be associated with multiple clients.) In addition to holding password shares, these servers may also be provisioned with arbitrary other information (that need not be stored by $C$ ). Any such information is provisioned by some incorruptible, central mechanism (a system administrator, say) at the outset of the protocol. This does not represent a restriction in practice, since the servers must be provisioned with correct password shares anyway, and so any additional information can be provided to the servers at that time. Furthermore, the servers have no restriction — as the client does — on the amount of 
information they can store. An (honest) execution of a protocol between client $C$ and associated servers $A$ and $B$ should result in the client holding two (independent) session keys $\mathrm{sk}_{C, A}, \mathrm{sk}_{C, B}$, and servers $A$ and $B$ holding sk ${ }_{A, C}$ and $\mathbf{s k}_{B, C}$, respectively, with $\mathrm{sk}_{C, A}=\mathrm{sk}_{A, C}$ and $\mathrm{sk}_{C, B}=\mathrm{sk}_{B, C}$.

\subsection{Passive Adversaries}

We assume an adversary who corrupts some servers at the outset of the protocol, such that for any client $C$ at most one of the servers associated with $C$ is corrupted. (Our definition does not require the adversary to corrupt one server associated with each client; thus, our definition encompasses security in the case when neither server associated with some client is corrupted.) In the case of a passive adversary, a corrupted server continues to operate according to the protocol but the adversary may monitor the corrupted server's internal state.

There are two types of communication: between clients and servers, and between servers. We give the adversary full control over the client-server communication; thus, the adversary can eavesdrop on this communication, send messages of its choice to servers or clients, or tamper with, delay, refuse to deliver, etc. any messages sent between servers and clients. Server-server communication is assumed to be done over a secure channel (this can be realized via standard use of private-key cryptography, since the servers can store long-term, shared keys); thus, the adversary is unable to eavesdrop on the communication between two uncorrupted servers, and communication between any two servers is not under adversarial control. ${ }^{3}$

With the above in mind, we proceed to the formal definitions.

Participants, passwords, and initialization. There is a fixed set of protocol participants (also called principals), each of which is either a client $C \in$ Client or a server $S \in$ Server, where Client and Server are disjoint. Each $C \in$ Client is assumed to have a password $p w_{C}$ chosen uniformly and independently from the "dictionary" $\{1, \ldots, N\}{ }^{4}$ As noted earlier, we make the simplifying assumption that each client shares its password with exactly two servers. If client $C$ shares its password with the distinct servers $A, B$, then $A$ (resp., $B$ ) holds a password share $p w_{C, A}$ (resp., $\left.p w_{C, B}\right)$; the mechanism for generating these shares depends on the protocol itself. We also allow each server to hold information in addition to these password shares. The initialization phase during which this information is provisioned is assumed to be carried out by some trusted authority, but any information stored by a corrupted server is available to the adversary.

In general, additional information can be generated during the initialization phase. For example, in the "hybrid" password/PKI model [22,7] public/secret key pairs are generated for each server and the secret key is given as input to the appropriate server, while the public key is provided to the appropriate client(s). For the protocol presented here, we require only the weaker requirement of a single set of public parameters that is provided to all parties.

Execution of the protocol. In the real world, a protocol determines how principals behave in response to messages from the network. In the formal model, these messages are provided by the adversary. Each principal is assumed to be able to execute the protocol multiple times

\footnotetext{
${ }^{3}$ For two uncorrupted servers, this follows by our assumption of a secure channel between them. A corrupted server in the passive setting communicates as specified by the protocol, and so we do not allow the adversary to control this communication. A corrupted server in the active setting may send whatever it likes and so it is unnecessary to allow the adversary to interfere with the communication channel in this case.

${ }^{4} \mathrm{As}$ in other work, our protocol and proof of security may be adapted easily to handle arbitrary dictionaries and/or non-uniform (but efficiently sampleable) distributions on passwords.
} 
(possibly concurrently) with different partners; this is modeled by allowing each principal to have an unlimited number of instances $[4,1]$ with which to execute the protocol. We denote instance $i$ of principal $U$ as $\Pi_{U}^{i}$. A given instance may be used only once. The adversary is given oracle access to these different instances, and each instance $\Pi_{U}^{i}$ maintains (local) state, updated during the course of the experiment, which includes the following:

- $\operatorname{sid}_{U}^{i}, \operatorname{pid}_{U}^{i}$, and $\mathrm{sk}_{U}^{i}$ are the session $i d$, partner $i d$, and session key(s) for an instance, respectively. The session id keeps track of the different executions of a particular user $U$; we specify below how this is determined. The partner id denotes the identity of the principal with whom $\Pi_{U}^{i}$ believes it is interacting. A client's partner id will be a set of two servers; a server's partner id will be a single client (viewed as a set for notational convenience). For $C$ a client, $\mathrm{sk}_{C}^{i}$ consists of a pair $\mathrm{sk}_{C, A}^{i}, \mathrm{sk}_{C, B}^{i}$, where these are the keys shared with servers $A$ and $B$, respectively. A server instance $\prod_{S}^{i}$ with partner $C$ has only a single session key sk ${ }_{S, C}^{i}$.

- $\operatorname{term}_{U}^{i}$ and $\operatorname{acc}_{U}^{i}$ are boolean variables indicating whether a given instance has terminated or accepted, respectively. Termination means that a given instance is done sending and receiving messages; acceptance indicates successful termination.

As highlighted earlier, the adversary is assumed to have complete control over all communication between the servers and clients. This is modeled via access to oracles which are essentially as in [1] and are described now:

- Send $(C, i, S$, msg), where $C \in$ Client and $S$ is a server associated with $C$. This sends message msg to client instance $\Pi_{C}^{i}$, supposedly from $S$. This client instance runs according to the protocol specification and the message it outputs, if any, is given to the adversary. If msg is empty then this query represents a "prompt" for $C$ to initiate the protocol.

- Send $(S, i, C$, msg), where $S \in$ Server is associated with client $C$. This sends message msg to server instance $\Pi_{S}^{i}$, supposedly from $C$. This instance runs according to the protocol specification, and the message it outputs is given to the adversary. If $S$ is corrupted, the adversary also receives the entire internal state of $S$; if the other server $S^{\prime}$ associated with $C$ is corrupted, then the adversary is given the entire internal state of $S^{\prime}$.

- Execute $(C, i, A, B, j)$, where $C \in$ Client and $A, B$ are servers associated with $C$. This executes the full protocol between instances $\Pi_{C}^{i}$ and $\Pi_{A}^{j}, \Pi_{B}^{j}$, and outputs the entire transcript of the client-server communication in this execution. In addition, if $S \in\{A, B\}$ is corrupted the adversary is given the entire internal state of $S$.

- Reveal $\left(U, U^{\prime}, i\right)$, where $U^{\prime} \in \operatorname{pid}_{U}^{i}$. This outputs $s_{U, U^{\prime}}^{i}$, a session key held by instance $\Pi_{U}^{i}$. This oracle call models usage of the session key by some higher-level application, or possible leakage of a session key due to, e.g., compromise of a host computer.

- Test $\left(U, U^{\prime}, i\right)$. This does not model any real capability of the adversary, but is instead needed for a definition of security. As in the case of a Reveal query, we assume $U^{\prime} \in \operatorname{pid}_{U}^{i}$. If $\mathrm{sk}_{U, U^{\prime}}^{i}=$ NULL, then this oracle outputs $\perp$. Otherwise, a random bit $b$ is generated; if $b=1$ the adversary is given $s_{U, U^{\prime}}^{i}$, and if $b=0$ the adversary is given a random session key. The adversary is allowed only a single Test query, at any time during its execution. 
As usual, Send oracle calls are intended to model active attacks on the protocol (i.e., "on-line attacks"), whereas Execute calls are intended to model passive eavesdropping (i.e., "off-line attacks").

Session ids and partnering. We define a natural notion of partnering based on matching transcripts. For a client instance $\Pi_{C}^{i}$ associated with servers $A$ and $B$, let $\operatorname{sid}_{C, A}^{i}$ (resp., $\operatorname{sid}_{C, B}^{i}$ ) denote the ordered sequence of messages sent to/from the client and server $A$ (resp., server $B$ ). For a server instance $\Pi_{B}^{j}$, let $\operatorname{sid}_{B}^{j}$ be the ordered sequence of messages sent to/from this instance and the client; note that server-server communication is not included. Then instances $\Pi_{C}^{i}$ and $\Pi_{B}^{j}$ are partnered if: (1) $\operatorname{sid}_{C, B}^{i}=\operatorname{sid}_{B}^{j} \neq \mathrm{NULL}$; and (2) $B \in \operatorname{pid}_{C}^{i}$ and $C \in \operatorname{pid}_{B}^{j}$.

Correctness. We require a key-exchange protocol to satisfy the following: if a client instance $\Pi_{C}^{i}$ and server instances $\Pi_{A}^{j}$ and $\Pi_{B}^{j}$ run an honest execution of the protocol with no interference from the adversary, then $\operatorname{acc}_{C}^{i}=\operatorname{acc}_{A}^{j}=\operatorname{acc}_{B}^{j}=$ TRUE, and $\mathrm{sk}_{C, A}^{i}=\mathrm{sk}_{A, C}^{j}$, and $\mathrm{sk}_{C, B}^{i}=\mathrm{sk}_{B, C}^{j}$.

In the case of an active adversary who has corrupted server $A$, we impose no correctness requirements. However, our protocol in Section 5 achieves the following: with all but negligible probability, for every pair of partnered instances $\Pi_{C}^{i}$ and $\Pi_{B}^{j}$ with $\operatorname{acc}_{C}^{i}=\operatorname{acc}_{B}^{j}=$ TRUE, it holds that $\mathrm{sk}_{C, B}^{i}=\mathrm{sk}_{B, C}^{j} \neq \mathrm{NULL}$.

Freshness. To formally define the adversary's success we must first define a notion of freshness for a session key, where freshness of a key is meant to indicate that the adversary does not trivially know the value of the key. We say a session key $s k_{U, U^{\prime}}^{i}$ is fresh if: (1) neither $U$ nor $U^{\prime}$ is a corrupted server, (2) the adversary never queried Reveal $\left(U, U^{\prime}, i\right)$; and (3) the adversary never queried Reveal $\left(U^{\prime}, U, j\right)$, where $\Pi_{U^{\prime}}^{j}$ and $\Pi_{U}^{i}$ are partnered.

Advantage of the adversary. Informally, the adversary succeeds if it can distinguish a fresh session key from random. (Restricting to a fresh session key is necessary for a meaningful definition of security.) Formally, we say an adversary $\mathcal{A}$ succeeds if it makes a single query Test $\left(U, U^{\prime}, i\right)$ regarding a fresh key $\mathrm{sk}_{U, U^{\prime}}^{i}$, and outputs a bit $b^{\prime}$ with $b^{\prime}=b$ (recall that $b$ is the bit chosen by the Test oracle). We denote this event by Succ. The advantage of adversary $\mathcal{A}$ in attacking protocol $P$ is then given by:

$$
\operatorname{Adv}_{\mathcal{A}, P}(k) \stackrel{\text { def }}{=} 2 \cdot \operatorname{Pr}[\text { Succ }]-1,
$$

where the probability is taken over the random coins used by the adversary as well as the random coins used during the course of the experiment.

An adversary can always succeed by trying all passwords one-by-one in an on-line impersonation attack; a protocol is secure if this is the best an adversary can do. In our model, on-line attacks correspond to Send queries. Formally, every instance for which the adversary has made a Send query counts as one on-line attack. We stress that instances with which the adversary interacts via Execute queries are not counted as on-line attacks. The number of on-line attacks represents a bound on the number of passwords the adversary could have tested in an on-line fashion.

Definition 1 Protocol $P$ is a secure two-server protocol for password-only authenticated key-exchange if, for all dictionary sizes $N$ and for all PPT adversaries $\mathcal{A}$ making at most $Q(k)$ on-line attacks and corrupting at most one server associated with each client, there exists a negligible function $\varepsilon(\cdot)$ such that $\operatorname{Adv}_{\mathcal{A}, P}(k) \leq Q(k) / N+\varepsilon(k)$.

Explicit mutual authentication. The above definition captures the requirement of implicit authentication only (and the protocol we present here achieves only implicit authentication). Using standard techniques, however, it is easy to add explicit authentication to any protocol achieving implicit authentication. 


\subsection{Active Adversaries}

The only difference in the active case is that the adversary may now cause any corrupted servers to deviate in an arbitrary way from the actions prescribed by the protocol. Thus, if a server is corrupted the adversary controls all messages sent from this server to any other servers. (The adversary can also control messages sent from this server to any clients; note, however, that even a passive adversary has this ability since it controls the communication channel between servers and clients.) Because of this change, we no longer use a Send oracle to model sending messages to a corrupted server, but we formally introduce a new Send oracle to model communication from a corrupted server to a non-corrupted server. (We also charge the adversary with an additional on-line attack for using the latter.) As in the passive case, however, we continue to assume that the adversary cannot eavesdrop on or control communication between two non-corrupted servers.

\section{A Review of the KOY Protocol}

Here, we provide a brief review of the KOY protocol [26] which will be useful toward understanding our two-server protocol in the following section. We also discuss the modifications we introduce to the KOY protocol which have the effect of both simplifying our eventual two-server protocol as well as improving the efficiency of the KOY protocol even in the single-server setting.

The KOY protocol operates in three rounds. At a high level, in round 1 the client generates a Cramer-Shoup encryption [14] of its password (using a "public key" contained in the public parameters) and sends the resulting ciphertext to the server; the server acts symmetrically in round 2. We observe that the following modifications may be made without affecting security of the protocol:

- The server may use El Gamal encryption [17] in round 2 rather than Cramer-Shoup encryption (this was first observed, in a different context, in [11]). This is somewhat surprising, as the proof of security in [26] explicitly relies on the chosen-ciphertext security of Cramer-Shoup encryption, while El Gamal encryption is only semantically secure.

- There is no need for the server to compute a fresh encryption of the password (in round 2) in each execution of the protocol; instead, the server can store an encryption of the password (along with the randomness used to generate this encryption) as part of its long-term state, and use the same ciphertext every time it executes the protocol.

We refer to the KOY protocol with the above modifications as the KOY* protocol. (We do not formally prove security of the $\mathrm{KOY}^{*}$ protocol in the single-server case; however, such a proof may be derived from the proofs of the two-server protocols that we give in this paper.)

In brief (we provide more detail when we discuss the two-server version of this protocol in the following section), then, the $\mathrm{KOY}^{*}$ protocol assumes public parameters containing a description of a group $\mathbb{G}$ with specified prime order $q$. Additionally, the parameters include random generators $g_{1}, g_{2}, g_{3}, h, c, d \in \mathbb{G}^{\times}$(where $\mathbb{G}^{\times} \stackrel{\text { def }}{=} \mathbb{G} \backslash\{1\}$ ) and a hash function $H$. Components $\left\langle g_{1}, g_{2}, h, c, d, H\right\rangle$ will be used for Cramer-Shoup encryption by the client in round 1 (as in the original KOY protocol), while $\left\langle g_{1}, g_{3}\right\rangle$ will be used for El Gamal encryption by the server in round 2 .

A password $p w_{C} \in \mathbb{Z}_{q}$ is chosen for each client $C$ and shared with the appropriate server $S$. In addition, $S$ is pre-provisioned with both $r^{\prime}$ and a ciphertext $\operatorname{Com}_{S, C} \stackrel{\text { def }}{=}\left(g_{1}^{r^{\prime}}, g_{3}^{r^{\prime}} g_{1}^{p w_{C}}\right)$, where $r^{\prime} \in \mathbb{Z}_{q}$ is chosen at random; note that Com ${ }_{S, C}$ is simply a (random) El Gamal encryption of $g_{1}^{p w_{C}}$. 
Execution of the protocol proceeds as follows. When a client Client with password $p w_{C}$ wants to initiate an execution of the protocol, it runs a key-generation algorithm for a one-time signature scheme, yielding $\mathrm{VK}$ and SK. It then chooses a random $r \in \mathbb{Z}_{q}$ and computes $A=g_{1}^{r}, B=g_{2}^{r}$, and $C=h^{r} \cdot g_{1}^{p w_{C}}$. It then computes $\alpha=H($ Client $|\mathrm{VK}| A|B| C)$ and sets $D=\left(c d^{\alpha}\right)^{r}$. Note that $(A, B, C, D)$ is a (labeled) Cramer-Shoup encryption of $g_{1}^{p w_{C}}$. The client sends

$$
\mathrm{msg}_{1} \stackrel{\text { def }}{=}\langle\text { Client, } \mathrm{VK}, A, B, C, D\rangle
$$

to the server.

The server re-computes $\alpha=H($ Client $|\mathrm{VK}| A|B| C)$, chooses random $x^{\prime}, y^{\prime}, z^{\prime}, w^{\prime} \in \mathbb{Z}_{q}$, and computes $E=g_{1}^{x^{\prime}} g_{2}^{y^{\prime}} h^{z^{\prime}}\left(c d^{\alpha}\right)^{w^{\prime}}$. It then sends the message

$$
\mathrm{msg}_{2} \stackrel{\text { def }}{=}\langle E, F, G\rangle
$$

to the client, where $(F, G)=\operatorname{Com}_{S, C}$. We stress that the same values $F, G$ are used by this server every time.

Upon receiving $\mathrm{msg}_{2}$, the client chooses random $x, y \in \mathbb{Z}_{q}$ and computes $K=g_{1}^{x} g_{3}^{y}$. It also computes a signature $\sigma$ on $\left(\mathrm{msg}_{1}, \mathrm{msg}_{2}, K\right)$ using SK, and sends

$$
\mathrm{msg}_{3} \stackrel{\text { def }}{=}\langle K, \sigma\rangle
$$

to the server. The client concludes by computing its session key as $\mathrm{sk}_{C}=E^{r} F^{x}\left(G / g_{1}^{p w_{C}}\right)^{y}$.

The server, upon receiving $\mathrm{msg}_{3}$, verifies the signature and aborts if it is incorrect. Otherwise, is computes its session key as $\mathrm{sk}_{S}=A^{x^{\prime}} B^{y^{\prime}}\left(C / g_{1}^{p w_{C}}\right)^{z^{\prime}} D^{w^{\prime}} K^{r^{\prime}}$. It may be easily verified that in a correct execution the client and server compute identical session keys.

\section{A Protocol Secure Against Passive Adversaries}

\subsection{Description of the Protocol}

We assume the reader is familiar with the decisional Diffie-Hellman (DDH) assumption [15], strong ${ }^{5}$ one-time signature schemes, and the Cramer-Shoup encryption scheme [14] with labels. A highlevel depiction of the protocol is given in Figures 1-3, and a more detailed description, as well as some informal discussion about the protocol, follows.

Initialization. During the initialization phase, public parameters (i.e., a common reference string) are generated and made available to all parties. For security parameter $k$, the public parameters for our protocol contain a group $\mathbb{G}$ (written multiplicatively) having specified prime order $q$ with $|q|=k$; we assume the hardness of the DDH problem in $\mathbb{G}$. Additionally, the parameters include random generators $g_{1}, g_{2}, g_{3}, h, c, d \in \mathbb{G} \backslash\{1\}$ and a hash function $H:\{0,1\}^{*} \rightarrow \mathbb{Z}_{q}$ chosen at random from a collision-resistant hash family.

As part of the initialization, each server $S$ is provisioned with an El Gamal public-/secret-key pair $\left(p k_{S}, s k_{S}\right)$, where $p k_{S}=g_{1}^{s k_{S}}$. If $A$ and $B$ are associated with the same client $C$, then $A$ (resp., $B$ ) is given $p k_{B}$ (resp., $p k_{A}$ ). We stress that, in contrast to the PKI-based model, the client is not assumed or required to know the public keys of any of the servers.

\footnotetext{
${ }^{5}$ In a strong signature scheme, an adversary cannot even forge a new signature on a previously signed message.
} 


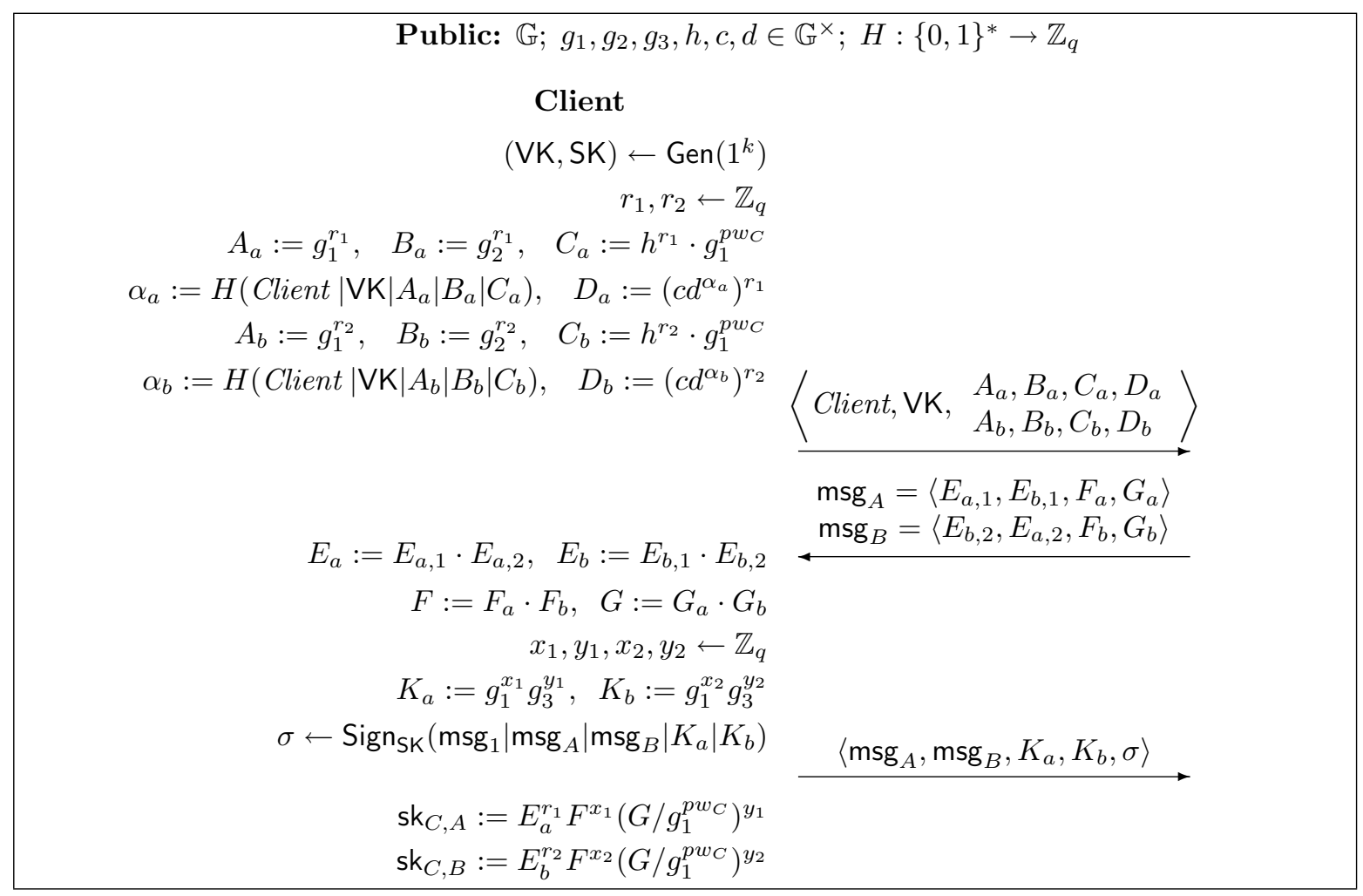

Figure 1: An execution of the protocol from the client's point of view.

Given a message $m \in \mathbb{G}$ and a public key (i.e., group element) $p k$, we let $M \leftarrow \operatorname{EIG}_{p k}(m)$ denote the act of choosing a random $r \in \mathbb{Z}_{q}$ and setting $M=\left(g_{1}^{r}, p k^{r} m\right)$. We let $M[1]$ refer to the first component of this ciphertext, and let $M[2]$ refer to the second. Note that if $s k$ is the corresponding secret key (i.e., $p k=g_{1}^{s k}$ ), then we have $m=\frac{M[2]}{M[1]^{s k}}$.

Passwords and password shares are provisioned in the following way: a password $p w_{C}$ is chosen randomly for each client $C$ and we assume that this password can be mapped in a one-to-one fashion to $\mathbb{Z}_{q}$. If $A$ and $B$ are the servers associated with a client $C$, then password shares $p w_{A, C}, p w_{B, C} \in \mathbb{Z}_{q}$ are chosen uniformly at random subject to $p w_{A, C}+p w_{B, C}=p w_{C} \bmod q$, with $p w_{A, C}$ given to server $A$ and $p w_{B, C}$ given to server $B$. In addition, both $A$ and $B$ are given $\operatorname{Com}_{A, C}, \operatorname{Com}_{A, C}^{\prime}, \operatorname{Com}_{B, C}$, and $\operatorname{Com}_{B, C}^{\prime}$, where:

$$
\begin{array}{lll}
\operatorname{Com}_{A, C} & \stackrel{\text { def }}{=} \operatorname{EIG}_{g_{3}}\left(g_{1}^{p w_{A, C}}\right)=\left(g_{1}^{r_{a}}, g_{3}^{r_{a}} g_{1}^{p w_{A, C}}\right) \\
\operatorname{Com}_{A, C}^{\prime} & \stackrel{\text { def }}{=} \operatorname{EIG}_{p k_{A}}\left(g_{1}^{p w_{A, C}}\right) \\
\operatorname{Com}_{B, C} & \stackrel{\text { def }}{=} & \operatorname{EIG}_{g_{3}}\left(g_{1}^{p w_{B, C}}\right)=\left(g_{1}^{r_{b}}, g_{3}^{r_{b}} g_{1}^{p w_{B, C}}\right) \\
\mathrm{Com}_{B, C}^{\prime} & \stackrel{\text { def }}{=} & \mathrm{EIG}_{p k_{B}}\left(g_{1}^{p w_{B, C}}\right) .
\end{array}
$$

(Note that the keys for these El Gamal encryptions differ.) Server $A$ (resp., server $B$ ) is additionally given the randomness $r_{a}$ (resp., $r_{b}$ ) used to construct $\operatorname{Com}_{A, C}\left(\operatorname{resp} ., \operatorname{Com}_{B, C}\right)$.

Protocol execution. At a high level one can view our protocol as two executions of the KOY* 
protocol, one between the client and server $A$ (using server $B$ to assist with the authentication), and one between the client and server $B$ (using server $A$ to assist with the authentication).

When a client with password $p w_{C}$ wants to initiate an execution of the protocol, this client computes Cramer-Shoup "encryptions" of $p w_{C}$ for each of the two servers. In more detail (cf. Figure 1), the client begins by running a key-generation algorithm for a one-time signature scheme, yielding verification key VK and signing key SK. The client next chooses random $r_{1} \in \mathbb{Z}_{q}$ and computes $A_{a}=g_{1}^{r_{1}}, B_{a}=g_{2}^{r_{1}}$, and $C_{a}=h^{r_{1}} \cdot g_{1}^{p w_{C}}$. The client then computes $\alpha_{a}=H\left(\right.$ Client $\left.|\mathrm{VK}| A_{a}\left|B_{a}\right| C_{a}\right)$ and sets $D=\left(c d^{\alpha_{a}}\right)^{r_{1}}$. This exact procedure is carried out a second time using an independent random value $r_{2} \in \mathbb{Z}_{q}$. The client sends

$$
\mathrm{msg}_{1} \stackrel{\text { def }}{=}\left\langle\text { Client, } \mathrm{VK}, A_{a}, B_{a}, C_{a}, D_{a}, A_{b}, B_{b}, C_{b}, D_{b}\right\rangle
$$

to each server as the first message of the protocol. Note that this corresponds to two independent "encryptions" of $p w_{C}$ using the label Client|VK.

The servers act symmetrically, so for simplicity we describe the actions of server $A$ (cf. Figure 2). Upon receiving $\mathrm{msg}_{1}$, server $A$ sends "shares" of (1) two values of the form $g_{1}^{x} g_{2}^{y} h^{z}\left(c d^{\alpha}\right)^{w}$ (for $\alpha \in\left\{\alpha_{a}, \alpha_{b}\right\}$ ), one for server $A$ and one for server $B$, and (2) an El Gamal encryption of $p w_{C}$. In more detail, server $A$ chooses random $x_{a}, y_{a}, z_{a}, w_{a} \in \mathbb{Z}_{q}$ and computes $E_{a, 1}=g_{1}^{x_{a}} g_{2}^{y_{a}} h^{z_{a}}\left(c d^{\alpha_{a}}\right)^{w_{a}}$. It also chooses random $x_{a}^{\prime}, y_{a}^{\prime}, z_{a}^{\prime}, w_{a}^{\prime} \in \mathbb{Z}_{q}$ and computes $E_{b, 1}=g_{1}^{x_{a}^{\prime}} g_{2}^{y_{a}^{\prime}} h^{z_{a}^{\prime}}\left(c d^{\alpha_{b}}\right)^{w_{a}^{\prime}}$. Finally, it sets $\left(F_{a}, G_{a}\right)$ equal to $\operatorname{Com}_{A, C}$ (which, recall, is an El Gamal encryption of $g_{1}^{p w_{A, C}}$ using "public key" $g_{3}$ and randomness $r_{a}$ ). It sends the message $\operatorname{msg}_{A} \stackrel{\text { def }}{=}\left\langle E_{a, 1}, E_{b, 1}, F_{a}, G_{a}\right\rangle$ to the client.

After receiving a second-round message from each server, the client combines the values thus received by multiplying them component-wise to obtain $\left\langle E_{a}, E_{b}, F, G\right\rangle$ (cf. Figure 1). Note that (1) neither server knows the representation of $E_{a}$ (resp., $E_{b}$ ) with respect to the basis $g_{1}, g_{2}, h,\left(c d^{\alpha_{a}}\right)$ (resp., $g_{1}, g_{2}, h,\left(c d^{\alpha_{b}}\right)$ ), and (2) the values $(F, G)$ form an El Gamal encryption of the client's password $p w_{C}$ (with respect to public key $g_{3}$ ). The client next chooses random values $x_{1}, y_{1}, x_{2}, y_{2} \in$ $\mathbb{Z}_{q}$, computes $K_{a}=g_{1}^{x_{1}} g_{3}^{y_{1}}$ and $K_{b}=g_{1}^{x_{2}} g_{3}^{y_{2}}$, and computes a signature $\sigma$ on msg $1\left|\mathrm{msg}_{A}\right| \mathrm{msg}_{B}\left|K_{a}\right| K_{b}$ using the secret key SK that it had previously generated. It sends $\mathrm{msg}_{3} \stackrel{\text { def }^{\prime}}{=}\left\langle\mathrm{msg}_{A}, \mathrm{msg}_{B}, K_{a}, K_{b}, \sigma\right\rangle$ to each server as the final message of the protocol. (Of course, $\operatorname{msg}_{A}$ need not be sent to $A$ and similarly for $\mathrm{msg}_{B}$. Furthermore, it would suffice for the client to sign and send hashes of these messages.) Finally, the client computes session keys

$$
\begin{aligned}
& \mathrm{sk}_{C, A}:=E_{a}^{r_{1}} F^{x_{1}}\left(G / g_{1}^{p w_{C}}\right)^{y_{1}} \\
& \text { sk }_{C, B}:=E_{b}^{r_{2}} F^{x_{2}}\left(G / g_{1}^{p w_{C}}\right)^{y_{2}} .
\end{aligned}
$$

Upon receiving $\left\langle\mathrm{msg}_{A}, \mathrm{msg}_{B}, K_{a}, K_{b}, \sigma\right\rangle$ from the client, server $A$ verifies that the $\left(F_{b}, G_{b}\right)$ component of $\operatorname{msg}_{B}$ is equal to $\operatorname{Com}_{B, C}$ (recall that $A$ stores $\operatorname{Com}_{B, C}$ ), and that $\sigma$ is a valid signature on msg $1\left|\mathrm{msg}_{A}\right| \mathrm{msg}_{B}\left|K_{a}\right| K_{b}$ with respect to VK. If verification fails, the server terminates the execution. Otherwise, servers $A$ and $B$ jointly execute the Compute protocol (cf. Figure 3 and described next) in order to compute their session keys.

Before describing the Compute protocol, we introduce notation for manipulation of El Gamal ciphertexts. If $M, M^{\prime}$ are two El Gamal ciphertexts (encrypted with respect to the same public key $p k)$, then we let $M \times M^{\prime}$ denote $\left(M[1] \cdot M^{\prime}[1], M[2] \cdot M^{\prime}[2]\right)$. Note that if $M$ is an encryption of $m$ and $M^{\prime}$ is an encryption of $m^{\prime}$, then $M \times M^{\prime}$ is an encryption of $m \cdot m^{\prime}$. For $x \in \mathbb{Z}_{q}$, we let $M^{x}$ denote the ciphertext $\left(M[1]^{x}, M[2]^{x}\right)$. Here, the resulting ciphertext is an encryption of $m^{x}$. 
Public: $\mathbb{G} ; g_{1}, g_{2}, g_{3}, h, c, d \in \mathbb{G}^{\times} ; H:\{0,1\}^{*} \rightarrow \mathbb{Z}_{q}$

\section{Client}

$\mathrm{msg}_{1}=\left\langle\right.$ Client $\left., \mathrm{VK}, \begin{array}{c}A_{a}, B_{a}, C_{a}, D_{a} \\ A_{b}, B_{b}, C_{b}, D_{b}\end{array}\right\rangle$

Server $A$

Compute $\alpha_{a}, \alpha_{b}$ as in Fig. 1

$x_{a}, y_{a}, z_{a}, w_{a} \longleftarrow \mathbb{Z}_{q}$

$x_{a}^{\prime}, y_{a}^{\prime}, z_{a}^{\prime}, w_{a}^{\prime} \longleftarrow \mathbb{Z}_{q}$

$E_{a, 1}:=g_{1}^{x_{a}} g_{2}^{y_{a}} h^{z_{a}}\left(c d^{\alpha_{a}}\right)^{w_{a}}$

$E_{b, 1}:=g_{1}^{x_{a}^{\prime}} g_{2}^{y_{a}^{\prime}} h^{z_{a}^{\prime}}\left(c d^{\alpha_{b}}\right)^{w_{a}^{\prime}}$

$\left(F_{a}, G_{a}\right):=\operatorname{Com}_{A, C}$

$$
\begin{gathered}
\stackrel{\left\langle E_{a, 1}, E_{b, 1}, F_{a}, G_{a}\right\rangle}{\longrightarrow} \\
\left\langle\mathrm{msg}_{A}, \mathrm{msg}_{B}, K_{a}, K_{b}, \sigma\right\rangle
\end{gathered}
$$

if $\left(F_{b}, G_{b}\right)$ incorrect, or

signature verification fails, output $\perp$

else: perform Compute to obtain $X_{a}$

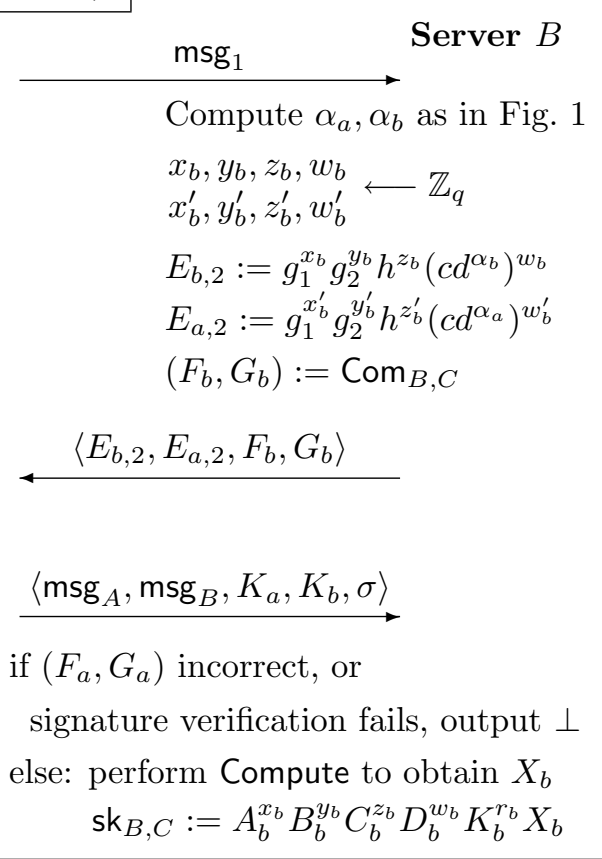

Figure 2: Execution of the protocol from the servers' points of view; see text for details. The Compute protocol is given in Figure 3.

With this in mind, we now describe the Compute protocol. Since the protocol is symmetric, we simply describe it from the point of view of server $A$. This server sets $M_{1}$ to be an El Gamal encryption (with respect to $p k_{A}$ ) of $g_{1}^{-z_{a}}$. It then sends $M_{1}$ to server $B$, who computes

$$
M_{2}^{\prime} \leftarrow M_{1}^{p w_{B, C}} \times\left(\mathrm{Com}_{A, C}^{\prime}\right)^{-z_{b}^{\prime}} \times \mathrm{EIG}_{p k_{A}}\left(g_{1}^{-z_{b}^{\prime} p w_{B, C}} A_{a}^{x_{b}^{\prime}} B_{a}^{y_{b}^{\prime}} C_{a}^{z_{b}^{\prime}} D_{a}^{w_{b}^{\prime}} K_{a}^{r_{b}}\right)
$$

and sends this value back to server $A$ (recall that $r_{b}$ is the randomness used to construct $\operatorname{Com}_{B, C}$ ). Finally, server $A$ decrypts $M_{2}^{\prime}$ and multiplies the result by $g_{1}^{-z_{a} \cdot p w_{A, C}}$ to obtain $X_{a}$. Note that

$$
X_{a}=g_{1}^{-\left(z_{a}+z_{b}^{\prime}\right) \cdot p w_{C}} \cdot\left(A_{a}^{x_{b}^{\prime}} B_{a}^{y_{b}^{\prime}} C_{a}^{z_{b}^{\prime}} D_{a}^{w_{b}^{\prime}} K_{a}^{r_{b}}\right),
$$

using the fact that $p w_{C}=p w_{A, C}+p w_{B, C} \bmod q$. In addition to the above, in the first step of the protocol the servers exchange the messages received from the client thus far; each server then verifies that these messages match their own view (and terminates if not).

Although omitted in the above description, we assume that the client and servers always verify that incoming messages are well-formed, and in particular that all appropriate components of the various messages indeed lie in $\mathbb{G}$ (we assume that membership in $\mathbb{G}$ can be efficiently verified).

Correctness. Since the protocol is symmetric, we focus on the session key shared between the 


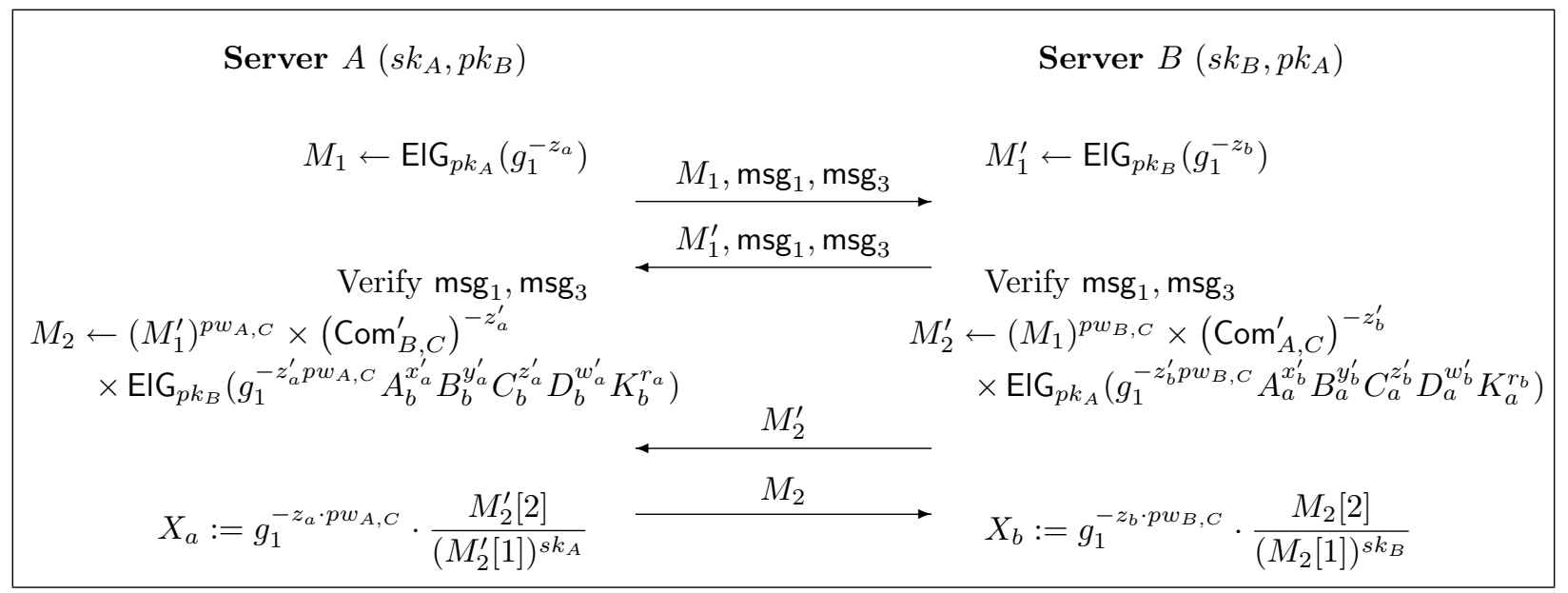

Figure 3: The Compute protocol. See text for a description of the notation used.

client and server $A$. The client computes:

$$
\begin{aligned}
\text { sk }_{C, A} & =E_{a}^{r_{1}} F^{x_{1}}\left(G / g_{1}^{p w_{C}}\right)^{y_{1}} \\
& =\left(g_{1}^{x_{a}+x_{b}^{\prime}} g_{2}^{y_{a}+y_{b}^{\prime}} h^{z_{a}+z_{b}^{\prime}}\left(c d^{\alpha_{a}}\right)^{w_{a}+w_{b}^{\prime}}\right)^{r_{1}} g_{1}^{\left(r_{a}+r_{b}\right) \cdot x_{1}} g_{3}^{\left(r_{a}+r_{b}\right) \cdot y_{1}} .
\end{aligned}
$$

Meanwhile, server $A$ computes:

$$
\begin{aligned}
\text { sk }_{A, C} & =A_{a}^{x_{a}} B_{a}^{y_{a}} C_{a}^{z_{a}} D_{a}^{w_{a}} K_{a}^{r_{a}} X_{a} \\
& =A_{a}^{x_{a}+x_{b}^{\prime}} B_{a}^{y_{a}+y_{b}^{\prime}} C_{a}^{z_{a}+z_{b}^{\prime}} D_{a}^{w_{a}+w_{b}^{\prime}} K_{a}^{r_{a}+r_{b}} \cdot g_{1}^{-\left(z_{a}+z_{b}^{\prime}\right) \cdot p w_{C}} \\
& =A_{a}^{x_{a}+x_{b}^{\prime}} B_{a}^{y_{a}+y_{b}^{\prime}}\left(C_{a} \cdot g_{1}^{-p w_{C}}\right)^{z_{a}+z_{b}^{\prime}} D_{a}^{w_{a}+w_{b}^{\prime}} K_{a}^{r_{a}+r_{b}},
\end{aligned}
$$

using Equation (1) to substitute for the value of $X_{a}$. Continuing, we have:

$$
\mathbf{s k}_{A, C}=\left(g_{1}^{x_{a}+x_{b}^{\prime}} g_{2}^{y_{a}+y_{b}^{\prime}} h^{z_{a}+z_{b}^{\prime}}\left(c d^{\alpha_{a}}\right)^{w_{a}+w_{b}^{\prime}}\right)^{r_{1}}\left(g_{1}^{x_{1}} g_{3}^{y_{1}}\right)^{r_{a}+r_{b}},
$$

which is equal to $\mathrm{sk}_{C, A}$.

Efficiency. We count exponentiations only, and assume a multi-exponentiation with up to 5 bases can be computed at the cost of at most 1.5 exponentiations. The client performs the equivalent of 15 full exponentiations (note that $p w_{C}$ is small, so does not count as a full exponentiation), while each server performs 13 exponentiations (computation of $M_{2}, M_{2}^{\prime}$ can be done by expressing each of them as the product of two multi-exponentiations). Thus, our protocol increases each party's computation relative to the original KOY protocol by about a factor of 2 . Performance could be further improved using pre-computation.

\subsection{Proof of Security for Passive Adversaries}

We prove the following theorem regarding the protocol of the previous section:

Theorem 1 Assuming (1) the DDH problem is hard for $\mathbb{G}$; (2) (Gen, Sign, Vrfy) is a strong onetime signature scheme; and (3) H is collision-resistant, the protocol of Figures 1-3 is a secure twoserver protocol for password-only authenticated key exchange in the presence of a passive adversary. 
Before proving this theorem, we introduce some notation. We view the adversary's queries to its Send oracles as queries to four different oracles $\operatorname{Send}_{0}$, Send ${ }_{1}, \mathrm{Send}_{2}$, Send ${ }_{3}$ corresponding, respectively, to the request for a client to initiate the protocol as well as the three rounds of the protocol. E.g., query $\operatorname{Send}_{0}(C, i, S, \varepsilon)$ represents a request for instance $\Pi_{C}^{i}$ of client $C$ to initiate the proto$\mathrm{col}$; the output of this query is an initial message $\left\langle\right.$ Client, $\left.\mathrm{VK}, A_{a}, \ldots, D_{a}, A_{b}, \ldots D_{b}\right\rangle$. Similarly, a query $\operatorname{Send}_{3}\left(A, i, C,\left\langle\mathrm{msg}_{A}, \mathrm{msg}_{B}, K_{a}, K_{b}, \sigma\right\rangle\right)$ represents sending message $\left\langle\mathrm{msg}_{A}, \mathrm{msg}_{B}, K_{a}, K_{b}, \sigma\right\rangle$ to instance $\Pi_{A}^{i}$ of server $A$. In the proof, we always let msg ${ }_{1}$ denote the initial message sent by the client to both servers; let $\mathrm{msg}_{A}$ (resp., $\mathrm{msg}_{B}$ ) denote the second-round message sent from server $A$ (resp., server $B$ ) to the client; and let $\mathrm{msg}_{3}$ denote the final message sent by the client.

We do not explicitly use collision-resistance of $H$ in the proof that follows, but that assumption is needed for security of the Cramer-Shoup encryption scheme (which is used in the proof).

Proof Given a passive adversary $\mathcal{A}$ attacking the protocol, we imagine a simulator that runs the protocol for $\mathcal{A}$. More precisely, after the adversary chooses which servers to corrupt, the simulator initializes the system (including selecting the public parameters, choosing passwords for all clients, and computing password shares - as well as any other necessary information - for all servers), gives $\mathcal{A}$ the information held by all corrupted servers, and then responds to the oracle calls made by $\mathcal{A}$. After computing the appropriate answer to the oracle query, the simulator provides the adversary with the internal state of any corrupted servers involved in the query.

When the adversary queries the Test oracle, the simulator chooses a random bit $b$. When the adversary completes its execution and outputs a bit $b^{\prime}$, the simulator can tell whether the adversary succeeds by checking whether (1) a single Test query was made regarding some fresh session key $\mathrm{sk}_{U, U^{\prime}}^{i}$, and (2) $b^{\prime}=b$. Success of the adversary is denoted by event Succ, and for any experiment $P$ we define $\operatorname{Adv}_{\mathcal{A}, P}(k) \stackrel{\text { def }}{=} 2 \cdot \operatorname{Pr}_{\mathcal{A}, P}[$ Succ $]-1$, where $\operatorname{Pr}_{\mathcal{A}, P}[\cdot]$ denotes the probability of an event when the simulator interacts with the adversary in accordance with experiment $P$. We refer to the original execution of the experiment (i.e., exactly according to the specification of the protocol) as $P_{0}$. We will introduce a sequence of transformations to the original experiment and bound the effect of each transformation on the adversary's advantage. We then bound the adversary's advantage in the final experiment; this yields a bound on the adversary's advantage in the original experiment.

It is useful to keep two observations in mind while reading the proof. First, the initial message of the Compute protocol (and the fact that we are in the passive setting) ensures that servers $A$ and $B$ abort without outputting a session key unless they have consistent views on the messages sent between the client and the servers. Second, when the two servers associated with some client are both uncorrupted the messages exchanged by the servers during the Compute protocol are irrelevant since the adversary cannot eavesdrop on their communication.

Experiment $P_{0}^{\prime}$ : In experiment $P_{0}^{\prime}$, the simulator interacts with the adversary as before except that the adversary does not succeed, and the experiment is aborted, if any of the following occur:

1. At any point, a msg ${ }_{1}$ generated by the simulator (whether in response to an Execute query or a Send 0 query) is repeated.

2. At any point, a msg ${ }_{A}$ or $\mathrm{msg}_{B}$ generated by the simulator (whether in response to an Execute query or a Send 1 query) is repeated.

3. At any point, the adversary forges a new, valid message/signature pair for any verification key used in a simulator-generated $\mathrm{msg}_{1}$ (whether $\mathrm{msg}_{1}$ was generated in response to an Execute query or a Send 0 query). 
In addition, the simulator changes how it computes the session key for any non-corrupted server $B$ so that it no longer uses the long-term El Gamal secret key $s k_{B}$. Recall that this secret key is used only to compute $X_{b}$ within the Compute protocol. Instead of computing $X_{b}$ this way, the simulator simply computes $X_{b}$ directly via:

$$
X_{b}:=g_{1}^{-\left(z_{b}+z_{a}^{\prime}\right) \cdot p w_{C}} \cdot\left(A_{b}^{x_{a}^{\prime}} B_{b}^{y_{a}^{\prime}} C_{b}^{z_{a}^{\prime}} D_{b}^{w_{a}^{\prime}} K_{b}^{r_{a}}\right) .
$$

We stress that the simulator can do this because we are dealing with a passive adversary (and so the simulator knows the internal state of all servers, even corrupted ones).

These changes have only a negligible effect on the adversary's advantage. Events 1 and 2, above, clearly occur with only negligible probability. Event 3 occurs with negligible probability assuming the security of (Gen, Sign, Vrfy) as a strong one-time signature scheme. Finally, the change in computing $X_{b}$ is merely a conceptual one since we assume a passive adversary (and so the value of $X_{b}$ is identical in games $P_{0}$ and $P_{0}^{\prime}$; cf. Equation 1). Putting everything together, we have that $\left|\operatorname{Adv}_{\mathcal{A}, P_{0}}(k)-\operatorname{Adv}_{\mathcal{A}, P_{0}^{\prime}}(k)\right|$ is negligible.

Experiment $P_{0}^{\prime \prime}$ : In this experiment, for any non-corrupted server $B$ the pre-provisioned value $\mathrm{Com}_{B, C}^{\prime}$ (for any client $C$ ) is set to be an El Gamal encryption of a random value (with respect to the public key $p k_{B}$ ). Similarly, when any non-corrupted server $B$ runs the Compute protocol with a corrupted server, $M_{1}^{\prime}$ is set to be an El Gamal encryption of a random value (with respect to the public key $p k_{B}$ ). Since, in experiment $P_{0}^{\prime}$, the simulator does not use the secret key $s k_{B}$ of any non-corrupted server $B$ (recall that computation of $X_{b}$ no longer uses $s k_{B}$ ), it follows from the semantic security of El Gamal encryption with respect to the public keys of all non-corrupted servers (as well as a straightforward hybrid argument) that $\left|\operatorname{Adv}_{\mathcal{A}, P_{0}^{\prime}}(k)-\operatorname{Adv}_{\mathcal{A}, P_{0}^{\prime \prime}}(k)\right|$ is negligible.

In the experiments that follow, we distinguish between the following possibilities for a msg ${ }_{1}$ received by a non-corrupted server: We say $\mathrm{msg}_{1}$ is oracle generated if it was output by the simulator in response to an oracle query (i.e., either an Execute query or a Send 0 query); we say $\mathrm{msg}_{1}$ is adversary generated otherwise. We also make the convention throughout the rest of the proof that any Send $_{3}$ queries made by the adversary contain a valid signature on the appropriate message as required by the protocol (since otherwise the server will simply reject the message and terminate the appropriate instance, and the adversary can determine by itself whether the signature is valid or not). Also, we assume that any components of adversary-generated messages that are supposed to lie in $\mathbb{G}$ actually do (as a server will again reject if this is not the case).

Experiment $P_{1}$ : In experiment $P_{1}$, the simulator changes the way it responds to a $\mathrm{msg}_{3}$ sent to an uncorrupted server, when $\mathrm{msg}_{1}$ for the corresponding server instance is oracle generated. (By definition, this is automatically the case for any Execute query being answered by the simulator.) Let $A$ and $B$ be the servers associated with some client $C$, and say $B$ is uncorrupted. Say a msg ${ }_{3}$ as described above is sent to instance $\Pi_{B}^{j}$, either as a result of an Execute $(C, i, A, B, j)$ query or a $\operatorname{Send}_{3}(B, j, C, \star)$ query, and that the corresponding instance of server $A$ has also been sent $\operatorname{msg}_{3}$. (If no instance of server $A$ has yet received the final message msg $_{3}$, then $\Pi_{B}^{j}$ will not execute beyond the first message of the Compute protocol.) The simulator searches for the unique $i$ such that $\operatorname{sid}_{C, B}^{i}=\operatorname{sid}_{B}^{j}$ (we comment below on the existence of such an $i$ ). The simulator then sets $\mathrm{sk}_{B, C}^{j}:=\mathrm{sk}_{C, B}^{i}$. Furthermore, if $A$ is corrupted then $M_{2}^{\prime}$ (in the Compute protocol) is computed as:

$$
M_{2}^{\prime} \leftarrow \mathrm{EIG}_{p k_{A}}\left(\mathrm{sk}_{C, A}^{i} \cdot A_{a}^{-x_{a}} B_{a}^{-y_{a}} C_{a}^{-z_{a}} D_{a}^{-w_{a}} K_{a}^{-r_{a}} g_{1}^{z_{a} \cdot p w_{A, C}}\right) .
$$


An $i$ as desired must exist, since otherwise the adversary has succeeded in forging a signature with respect to a verification key generated by the simulator and the simulator would then have aborted (cf. experiment $P_{0}^{\prime}$ ). Furthermore, this $i$ must be unique or else an oracle-generated msg ${ }_{1}$ has repeated and the simulator would have aborted.

The changes just described do not have any effect on the view of the adversary. This is clear for the case of $\mathrm{sk}_{B, C}^{j}$, since in $P_{0}^{\prime \prime}$ it is always the case that $\mathrm{sk}_{B, C}^{j}=\mathrm{sk}_{C, B}^{i}$ when $\Pi_{B}^{j}$ and $\Pi_{C}^{i}$ are partnered and $B$ does not terminate the protocol. (Recall we assume a passive adversary.) Furthermore, in both experiments $P_{1}$ and $P_{0}^{\prime \prime}$, the value $M_{2}^{\prime}$ is a random encryption of the unique value $v$ such that

$$
\mathrm{sk}_{A, C}^{j} \stackrel{\text { def }}{=} v \cdot\left(g_{1}^{-z_{a} \cdot p w_{A, C}} A_{a}^{x_{a}} B_{a}^{y_{a}} C_{a}^{z_{a}} D_{a}^{w_{a}} K_{a}^{r_{a}}\right)=\mathrm{sk}_{C, A}^{i} \cdot
$$

Thus, $\operatorname{Adv}_{\mathcal{A}, P_{1}}(k)=\operatorname{Adv}_{\mathcal{A}, P_{0}^{\prime \prime}}(k)$.

We next introduce another conceptual change in the experiment by having the simulator choose public parameters $h, c, d$ in such a way that it knows their representations with respect to $g_{1}, g_{2}$. That is, when generating the public parameters the simulator now chooses $g_{1}, g_{2} \leftarrow \mathbb{G} \backslash\{1\}$ (as before) and then chooses $\kappa \leftarrow \mathbb{Z}_{q}^{*}$ and $\left(\chi_{1}, \chi_{2}\right),\left(\xi_{1}, \xi_{2}\right) \leftarrow\left\{(x, y) \in \mathbb{Z}_{q} \times \mathbb{Z}_{q} \mid g_{1}^{x} g_{2}^{y} \neq 1\right\}$. It then sets

$$
h=g_{1}^{\kappa}, \quad c=g_{1}^{\chi_{1}} g_{2}^{\chi_{2}}, \quad d=g_{1}^{\xi_{1}} g_{2}^{\xi_{2}} .
$$

It is clear that the resulting distribution on the public parameters is unchanged from the previous experiment, and so this has no effect on the adversary's view.

Say (Client, VK, $A, B, C, D$ ) is a correct encryption if $A^{\chi_{1}+\alpha \xi_{1}} B^{\chi_{2}+\alpha \xi_{2}}=D$ and $C / A^{\kappa}=g_{1}^{p w_{C l i e n t}}$, where $\alpha \stackrel{\text { def }}{=} H($ Client $|\mathrm{VK}| A|B| C)$; otherwise, it is an incorrect encryption. We now distinguish three types of adversary-generated $\mathrm{msg}_{1}=\left\langle\right.$ Client, $\left.\mathrm{VK}, \begin{array}{c}A_{a}, B_{a}, C_{a}, D_{a} \\ A_{b}, B_{b}, C_{b}, D_{b}\end{array}\right\rangle$ :

- We say $\mathrm{msg}_{1}$ re-uses an oracle-generated verification key if $\mathrm{VK}$ is identical to a verification key used in an oracle-generated $\mathrm{msg}_{1}$.

- We say $\mathrm{msg}_{1}$ is valid if either (Client, $\left.\mathrm{VK}, A_{a}, B_{a}, C_{a}, D_{a}\right)$ or (Client, $\left.\mathrm{VK}, A_{b}, B_{b}, C_{b}, D_{b}\right)$ is a correct encryption, and $\mathrm{msg}_{1}$ does not re-use an oracle-generated verification key.

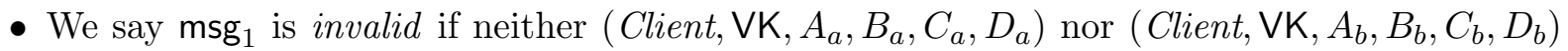
are correct encryptions, and $\mathrm{msg}_{1}$ does not re-use an oracle-generated verification key.

The simulator can always tell whether a given $\mathrm{msg}_{1}$ re-uses an oracle-generated verification key, and (due to the way the public parameters are now generated) can also efficiently determine whether a given $\mathrm{msg}_{1}$ is valid or invalid.

Experiment $P_{2}$ : In this experiment, the simulator changes the way it responds to a $\mathrm{msg}_{3}$ sent to an uncorrupted server when the $\mathrm{msg}_{1}$ for the corresponding server instance is adversary-generated and invalid. Let $A$ and $B$ be the servers associated with some client $C$, and say $B$ is uncorrupted. When a msg $_{3}$ as described above is sent to an instance $\Pi_{B}^{j}$ of server $B$, and $B$ does not terminate the protocol, then the session key $\mathrm{sk}_{B, C}^{j}$ is chosen uniformly at random from $\mathbb{G}$. Furthermore, $M_{2}^{\prime}$ (in the Compute protocol) is computed as an encryption (with respect to public key $p k_{A}$ ) of a uniform element of $\mathbb{G}$. We claim that:

Claim $1 \operatorname{Adv}_{\mathcal{A}, P_{2}}(k)=\operatorname{Adv}_{\mathcal{A}, P_{1}}(k)$. 
We prove this by showing that the distributions on the adversary's views in the two experiments are identical. Assume $A$ is corrupted, as this is the more difficult case. Let us focus first on the case of $\mathrm{sk}_{B, C}^{j}$. Say

$$
\mathrm{msg}_{1}=\left\langle\text { Client }, \mathrm{VK}, \begin{array}{c}
A_{a}, B_{a}, C_{a}, D_{a} \\
A_{b}, B_{b}, C_{b}, D_{b}
\end{array}\right\rangle
$$

where $\mathrm{msg}_{1}$ is the first message sent to the server instance under consideration. Since $\mathrm{msg}_{1}$ is invalid, we know that (Client, VK, $A_{b}, B_{b}, C_{b}, D_{b}$ ) is an incorrect encryption. So, it must be the case that either $A_{b}^{\chi_{1}+\alpha_{b} \xi_{1}} B_{b}^{\chi_{2}+\alpha_{b} \xi_{2}} \neq D_{b}$ or $C_{b} / p w_{\text {Client }} \neq A_{b}^{\kappa}$ (or possibly both). In either case, $\left(A_{b}, B_{b}, C_{b} / p w_{\text {Client }}, D_{b}\right)$ is not a DDH tuple with respect to the basis $\left(g_{1}, g_{2}, h, c d^{\alpha_{b}}\right)$. Thus, for any $\mu, \nu \in \mathbb{G}$ and fixing the randomness used in the rest of experiment $P_{1}$, the probability over choice of $x_{b}, y_{b}, z_{b}, w_{b}$ that $E_{b, 2}=\mu$ and $\mathrm{sk}_{B, C}^{j}=\nu$ is exactly the probability that

$$
\log \mu=x_{b}+y_{b} \cdot \log g_{2}+z_{b} \cdot \log h+w_{b} \cdot \log \left(c d^{\alpha_{b}}\right)
$$

and

$$
\begin{aligned}
& \log \nu-\left(r_{a}+r_{b}\right) \cdot \log K_{b}= \\
& \quad\left(x_{b}+x_{a}^{\prime}\right) \cdot \log A_{b}+\left(y_{b}+y_{a}^{\prime}\right) \cdot \log B_{b}+\left(z_{b}+z_{a}^{\prime}\right) \cdot \log \left(C_{b} / g_{1}^{p w_{C}}\right)+\left(w_{b}+w_{a}^{\prime}\right) \cdot \log D_{b},
\end{aligned}
$$

where all logarithms are with respect to the base $g_{1}$, and $p w_{C}=p w_{\text {Client }}$. Note that $x_{a}^{\prime}, y_{a}^{\prime}, z_{a}^{\prime}, w_{a}^{\prime}$ are independent of $x_{b}, y_{b}, z_{b}, w_{b}$ since we assume a passive adversary. As in [26], then, it can be verified that Equations (2) and (3) are linearly independent and not identically zero, when viewed as equations over $\mathbb{Z}_{q}$ in the variables $x_{b}, y_{b}, z_{b}, w_{b} .{ }^{6}$ Thus, the desired probability is exactly $1 / q^{2}$ and hence the value of $\mathbf{s k}_{B, C}^{j}$ is uniformly distributed in $\mathbb{G}$ independent of the rest of the experiment.

A similar argument holds for the values of $M_{2}^{\prime}$ and $E_{a, 2}$, viewed as functions of the random variables $x_{b}^{\prime}, y_{b}^{\prime}, z_{b}^{\prime}, w_{b}^{\prime}$ and using now the fact that (Client, $\mathrm{VK}, A_{a}, B_{a}, C_{a}, D_{a}$ ) is an incorrect encryption. In particular, $\mathrm{Com}_{A, C}^{\prime}$ is an El Gamal encryption (with respect to $p k_{A}$ ) of $g_{1}^{p w_{A, C}}$ and hence (in experiment $\left.P_{1}\right) M_{2}^{\prime}$ is an El Gamal encryption of the value

$$
g_{1}^{-z_{a} \cdot p w_{B, C}} K_{a}^{r_{b}} \cdot\left(A_{a}^{x_{b}^{\prime}} B_{a}^{y_{b}^{\prime}}\left(C_{a} / g_{1}^{p w_{C}}\right)^{z_{b}^{\prime}} D_{a}^{w_{b}^{\prime}}\right) .
$$

The rest of the argument is exactly as above. This concludes the proof of the claim.

Experiment $P_{3}$ : Here, the simulator changes the way it responds to a $\mathrm{msg}_{3}$ being sent to an uncorrupted server when $\mathrm{msg}_{1}$ for the corresponding server instance is adversary-generated and valid. In this case, if msg $_{1}$ was also sent to the other server associated with the same client, the simulator halts and the adversary succeeds. (In any other case, the adversary's success is determined as in the previous experiment. Note also that in this experiment we no longer need to compute $X_{b}$ on behalf of a non-corrupted server, since that value is now no longer used.) Clearly, $\operatorname{Adv}_{\mathcal{A}, P_{2}}(k) \leq \operatorname{Adv}_{\mathcal{A}, P_{3}}(k)$ since there are now more ways for the adversary to succeed.

Experiment $P_{4}$ : Let us first summarize where things stand in experiment $P_{3}$. In that experiment, for any non-corrupted server $B$, the simulator does not use the value $r_{b}$ (i.e., the randomness used to construct $\left.\operatorname{Com}_{B, C}\right)$ at any point during the experiment. In particular, for a given instance $\Pi_{B}^{j}$ with partner $C$ :

\footnotetext{
${ }^{6}$ We use here the fact that $M_{1}^{\prime}$ is no longer an encryption of $z_{b}$ (cf. experiment $P_{0}^{\prime \prime}$ ) and hence the only information the adversary has about $x_{b}, y_{b}, z_{b}, w_{b}$ comes from $E_{b, 2}$ and $\mathbf{s k}_{B, C}$.
} 
- If $\mathrm{msg}_{1}$ sent to this instance is oracle generated, the simulator computes $\mathrm{sk}_{B, C}^{j}$ and $M_{2}^{\prime}$ as described in experiment $P_{1}$.

- If $\mathrm{msg}_{1}$ to this instance is adversary-generated and re-uses an oracle-generated verification key, the simulator aborts if the adversary later sends a $\mathrm{msg}_{3}$ to this instance which contains a valid signature.

- If $\mathrm{msg}_{1}$ to this instance is adversary-generated and invalid, the simulator chooses $\mathrm{sk}_{B, C}^{j}$ and $M_{2}^{\prime}$ at random, as described in experiment $P_{2}$.

- Finally, if $\mathrm{msg}_{1}$ to this instance is adversary-generated and valid, then the simulator halts and the adversary succeeds as described in experiment $P_{3}$.

Let $A$ and $B$ be servers associated with some client $C$, and say $B$ is non-corrupted. In experiment $P_{4}$, the simulator changes $\operatorname{Com}_{B, C}$. Whether $A$ is corrupted or not, $\operatorname{Com}_{A, C}$ is computed as before (namely, as a random encryption under public key $g_{3}$ of a random value $p w_{A, C}$ ). The simulator sets $\mathrm{Com}_{B, C}$ to be a random El Gamal encryption of $g_{1}^{N+1-p w_{A, C}}$ with respect to public key $g_{3}$. (Note that the pair $p w_{A, C}, N+1-p w_{A, C}$ is now a random sharing of the invalid password $N+1$. In particular, servers $A$ and $B$ are given identically distributed information.) It follows readily from the semantic security of El Gamal encryption that $\left|\operatorname{Adv}_{\mathcal{A}, P_{4}}-\operatorname{Adv}_{\mathcal{A}, P_{3}}\right|$ is negligible. We remark that in experiment $P_{4}$, the simulator never uses $p w_{C}$ (for any client $C$ ) in simulating the actions of any non-corrupted server $B$.

Before continuing, we introduce definitions for the messages received by the client in the second round of the protocol that are analogous to those given previously for the case of $\mathrm{msg}_{1}$. For a pair $\left(\mathrm{msg}_{A}, \mathrm{msg}_{B}\right)$ received by the client, say the pair is oracle generated if both $\mathrm{msg}_{A}$ and $\mathrm{msg}_{B}$ were output by the simulator in response to an oracle query (i.e., either an Execute query or a Send ${ }_{1}$ query). The pair is adversary generated otherwise.

We also introduce another conceptual change in the experiment by now having the simulator choose public parameter $g_{3}$ by first selecting random $\lambda \leftarrow \mathbb{Z}_{q}^{*}$ and then setting $g_{3}=g_{1}^{\lambda}$. It is clear that the resulting distribution on $g_{3}$ is unchanged. With this in place, we can now define notions of validity/invalidity in a way analogous to that done previously for the case of $\mathrm{msg}_{1}$. Namely, say a client receives messages $\mathrm{msg}_{A}=\left\langle E_{a, 1}, E_{b, 1}, F_{a}, G_{a}\right\rangle$ and $\mathrm{msg}_{B}=\left\langle E_{b, 2}, E_{a, 2}, F_{b}, G_{b}\right\rangle$, and define $E_{a}, E_{b}, F, G$ as in the client's execution. We say the pair $\left(\mathrm{msg}_{A}, \mathrm{msg}_{B}\right)$ is valid if $G / F^{\lambda}=g_{1}^{p w_{C}}$ and invalid otherwise. Since the simulator knows $\log _{g_{1}} g_{3}$, it can efficiently determine validity. We observe at this point that if both $\mathrm{msg}_{A}$ and $\mathrm{msg}_{B}$ are oracle generated, then $\left(\mathrm{msg}_{A}, \mathrm{msg}_{B}\right)$ is invalid because of the way $\operatorname{Com}_{A, C}$ and $\operatorname{Com}_{B, C}$ was computed in $P_{4}$.

Experiment $P_{5}$ : In experiment $P_{5}$, the simulator changes the way it responds to an invalid $\left(\mathrm{msg}_{A}, \mathrm{msg}_{B}\right)$. In that case, the simulator computes $\mathrm{msg}_{3}$ as in the previous experiment but then chooses keys $\mathrm{sk}_{C, A}$ and $\mathrm{sk}_{C, B}$ independently and uniformly at random from $\mathbb{G}$.

Claim $2 \operatorname{Adv}_{\mathcal{A}, P_{4}}(k)=\operatorname{Adv}_{\mathcal{A}, P_{5}}(k)$.

The proof of this claim follows that of Claim 1. We prove the claim by showing that the distributions on the adversary's views in the two experiments are identical. Let $\left\langle E_{a}, E_{b}, F, G\right\rangle$ be as computed by some client $C$ after receiving $\left(\mathrm{msg}_{A}, \mathrm{msg}_{B}\right)$. By definition of invalidity, we have that $G / F^{\lambda} \neq g_{1}^{p w_{C}}$, where $\lambda \stackrel{\text { def }}{=} \log _{g_{1}} g_{3}$. Letting $s=\log _{g_{1}} F$ and $s^{\prime}=\log _{g_{1}}\left(G / g_{1}^{p w_{c}}\right)$, this means that $s^{\prime} \neq \lambda s$. For any 
$\mu_{1}, \mu_{2}, \nu_{1}, \nu_{2} \in \mathbb{G}$, the probability over choice of $x_{1}, y_{1}, x_{2}, y_{2}$ (fixing the randomness used in the remainder of the experiment) that $K_{a}=\mu_{1}, K_{b}=\mu_{2}, \mathbf{s k}_{C, A}=\nu_{1}$, and $\mathbf{s k}_{C, B}=\nu_{2}$ is exactly the probability that the following linear equations in $x_{1}, y_{1}, x_{2}, y_{2}$ (over $\mathbb{Z}_{q}$ ) hold:

$$
\begin{aligned}
\log _{g_{1}} \mu_{1} & =x_{1}+\lambda \cdot y_{1} \\
\log _{g_{1}} \mu_{2} & =x_{2}+\lambda \cdot y_{2} \\
\log _{g_{1}} \nu_{1}-r_{1} \log _{g_{1}} E_{a} & =x_{1} \cdot s+y_{1} \cdot s^{\prime} \\
\log _{g_{1}} \nu_{2}-r_{2} \log _{g_{1}} E_{b} & =x_{2} \cdot s+y_{2} \cdot s^{\prime} .
\end{aligned}
$$

It is easy to see that these equations are linearly independent (given that $s^{\prime} \neq \lambda s$ ) and so the desired probability is $1 / q^{4}$. Hence, as desired, the values of $\mathbf{s k}_{C, A}$ and $\mathbf{s k}_{C, B}$ are uniformly and independently distributed.

Experiment $P_{6}$ : Paralleling the change made in experiment $P_{3}$, the simulator now changes the way it responds to a valid (adversary-generated) $\left(\mathrm{msg}_{A}, \mathrm{msg}_{B}\right)$ : upon receiving such a message the simulator halts and the adversary succeeds. (In any other case, the adversary succeeds as in the previous experiment.) Clearly, this can only increase the adversary's probability of success and so $\operatorname{Adv}_{\mathcal{A}, P_{5}}(k) \leq \operatorname{Adv}_{\mathcal{A}, P_{6}}(k)$.

Experiment $P_{7}$ : In experiment $P_{6}$, the simulator does not use the values $r_{1}, r_{2}$ (in simulating a client instance) other than to construct the Cramer-Shoup ciphertexts $\left(A_{a}, \ldots, D_{a}\right)$ and $\left(A_{b}, \ldots, D_{b}\right)$ which are encryptions of the correct password value $g_{1}^{p w_{C}}$. In experiment $P_{7}$, the simulator instead sets these ciphertexts to be encryptions of 1 (i.e., it sets $C_{a}=h^{r_{1}}$ and $C_{b}=h^{r_{2}}$ ). (Note this corresponds to the "password" 0.) The following bounds the effect this can have on the adversary's success probability:

Claim 3 Under the DDH assumption, $\left|\operatorname{Adv}_{\mathcal{A}, P_{6}}(k)-\operatorname{Adv}_{\mathcal{A}, P_{7}}(k)\right|$ is negligible.

The claim follows from the security of the Cramer-Shoup encryption scheme (with labels) under adaptive chosen-ciphertext attack. The proof follows that of [26], and we merely sketch an outline here. Given a public-key $\left(g_{1}, g_{2}, h, c, d\right)$ for an instance of the Cramer-Shoup encryption scheme, as well as access to an encryption oracle and a decryption oracle, the simulator will first use the given values as the appropriate portion of the public parameters. The remaining public parameter, $g_{3}$, is generated so that the simulator knows $\log _{g_{1}} g_{3}$; the distribution of $g_{3}$ is the same as in $P_{6}$. Every time the simulator responds to a request to initiate an interaction on behalf of a client $C$ (i.e., as a result of an Execute query or a Send 0 query), it generates a verification key VK as before and then submits (twice) to its encryption oracle the query ( $\langle$ Client, $\left.\mathrm{VK}), g_{1}^{p w_{C}}, 1\right)$ and receives in return ciphertexts $\left(A_{a}, B_{a}, C_{a}, D_{a}\right)$ and $\left(A_{b}, B_{b}, C_{b}, D_{b}\right)$. (In the query to the encryption oracle, $\langle$ Client, VK $\rangle$ is the label, and $g_{1}^{p w_{C}}$ and 1 are the two messages.) The simulator then uses these ciphertexts to construct $\mathrm{msg}_{1}$. As we have already noted, the remainder of the client-side interaction can be simulated with no difficulty. As for the server-side interactions, here the simulator needs to be able to determine whether an adversary-generated msg $_{1}$ is valid or not. It can do this using its decryption oracle, with the only subtlety being to verify that the simulator never needs to query the decryption oracle with a (label, ciphertext) pair it received from its encryption oracle. This is not quite as obvious in our case as in [26] because it is possible in our case for a $\mathrm{msg}_{1}$ to be adversarygenerated but for one of the component ciphertexts of this message to be equal to a component ciphertext of an oracle-generated $\mathrm{msg}_{1}$. Yet it remains true due to the following observations: 
- If $\mathrm{msg}_{1}$ is oracle generated, then no decryption is needed.

- If an adversary-generated $\mathrm{msg}_{1}$ re-uses an oracle-generated verification key, the simulator will abort if the adversary later sends a msg $_{3}$ with a valid signature (because this implies a signature forgery).

- Otherwise, the verification key VK used in $\mathrm{msg}_{1}$ is different from all oracle-generated verification keys, and so the (label, ciphertext) pair submitted by the simulator to its decryption oracle is different from all (label, ciphertext) pairs received from its encryption oracle.

The proof concludes by noting that when the encryption oracle encrypts its left-most input, the adversary's view is identical to its view in experiment $P_{6}$, while when the encryption oracle encrypts its right-most input, the adversary's view is identical to its view in experiment $P_{7}$.

The adversary's view in experiment $P_{7}$ is independent of the passwords chosen for the various clients except for the fact that the adversary learns whether adversary-generated $\mathrm{msg}_{1}$ or $\left(\mathrm{msg}_{A}, \mathrm{msg}_{B}\right)$ are valid or not. That is, although the adversary is given password shares $p w_{A, C}$ for any corrupted server(s) $A$ and any client(s) $C$ associated with $A$, these shares are uniformly distributed in $\mathbb{Z}_{q}$ and therefore contain no information about the actual client password(s) $p w_{C}$. Furthermore, the simulator does not use $p w_{C}$ except to test whether adversary-generated $\mathrm{msg}_{1}$ and $\left(\mathrm{msg}_{A}, \mathrm{msg}_{B}\right)$ are valid or not. Let GuessPWD denote the event that the adversary sends a valid $\mathrm{msg}_{1}$ or $\left(\mathrm{msg}_{A}, \mathrm{msg}_{B}\right)$. The probability of GuessPWD is at most $Q(k) / N$, where $Q(k)$ is the number of on-line attacks made by $\mathcal{A}$. (Although the adversary gets the equivalent of two password guesses for each $\mathrm{msg}_{1}$, for the adversary to succeed it must send a valid msg ${ }_{1}$ to both servers associated with some client. Thus, this requires two on-line attacks.) If GuessPWD does not occur then the adversary can succeed only by correctly guessing the value of $b$ used by the Test oracle. In this case, however, all fresh session keys are uniformly distributed in $\mathbb{G}$ independent of the adversary's view, and so the probability that the adversary can correctly guess $b$ in this case is exactly $1 / 2$.

Putting everything together, we have:

$$
\begin{aligned}
\operatorname{Pr}_{\mathcal{A}, P_{7}}[\text { Succ }] & =\operatorname{Pr}[\text { GuessPWD }]+\frac{1}{2} \cdot(1-\operatorname{Pr}[\text { GuessPWD }]) \\
& =\frac{1}{2} \cdot \operatorname{Pr}[\text { GuessPWD }]+\frac{1}{2} \\
& \leq \frac{Q(k)}{2 N}+\frac{1}{2}
\end{aligned}
$$

and thus the adversary's advantage in experiment $P_{7}$ is at most $Q(k) / N$. The sequence of claims proven above show that

$$
\operatorname{Adv}_{\mathcal{A}, P_{0}}(k) \leq \operatorname{Adv}_{\mathcal{A}, P_{7}}(k)+\varepsilon(k)
$$

for some negligible function $\varepsilon(\cdot)$, and therefore the adversary's advantage in $P_{0}$ (i.e., the original protocol) is at most $Q(k) / N$ plus some negligible quantity, as desired.

\section{$5 \quad$ Handling Active Adversaries}

Here, we describe the necessary changes to the protocol in order to handle active adversaries. We then sketch the appropriate modifications to the proof given in the previous section. 


\subsection{Overview of Changes to the Protocol}

At a high level, the changes we make can be summarized as follows:

Proofs of correctness. We require servers to give proofs of correctness for their actions during the Compute protocol. We stress that we use only the fact that these are proofs (and not proofs of knowledge) and therefore we do not require any rewinding in our proof of security. This is crucial, as it enables us to handle concurrent executions of the protocol. Nevertheless, as part of the proofs of correctness we will have the servers encrypt certain values with respect to (additional) per-server public keys provisioned during protocol initialization. This will, in fact, enable extraction of certain values from the adversary during the security proof.

Commitments to password shares. The protocol as described in the previous section already assumes that each server is provisioned with appropriate El Gamal encryptions of the password share of the other server. We will use these encrypted values (along with the proofs of correctness discussed earlier) to "force" a corrupted server to use the correct password share in its computations.

Simulating proofs for non-corrupted servers. During the course of the proof of security it will be necessary for non-corrupted servers to deviate from the prescribed actions of the protocol, yet these servers must give "valid" proofs of correctness to corrupted servers. We cannot use "standard" zero-knowledge proofs in our setting, since (1) this would require rewinding which we explicitly want to avoid, and (2) potential malleability issues arise due to the fact that a corrupted server may be giving its proof of correctness at the same time a non-corrupted server is giving such a proof (this is so even if we force sequential executions of the proofs of correctness within any particular instance, since multiple instances may be simultaneously active). To enable simulatability in a concurrent setting we rely on techniques of MacKenzie [29] described in greater detail below.

\subsection{Detailed Description of Changes to the Protocol}

We first discuss the necessary modifications to the initialization phase. In addition to the values already discussed in Section 4.1: (1) each server $S$ is given a random triple triple $S_{S}=\left(U_{S, 1}, U_{S, 2}, U_{S, 3}\right)$ of elements chosen uniformly at random from $\mathbb{G}$. Furthermore, (2) if servers $A$ and $B$ are associated with the same client $C$, then $B$ is given triple $A$ and $A$ is given triple ${ }_{B}$.

We next describe the necessary changes to the protocol itself. In what follows, we use witnessindistinguishable $\Sigma$-protocols (with negligible soundness error ${ }^{7}$ ) [12] of various predicates and it will be useful to develop some notation. If $\Psi$ represents a predicate (defined over some public values), we let $\Sigma[\Psi]$ denote a $\Sigma$-protocol for this predicate. If $\Psi_{1}, \Psi_{2}$ are two predicates, then we let $\Sigma\left[\Psi_{1} \vee \Psi_{2}\right]$ denote a $\Sigma$-protocol for the "or" of these predicates. Given $\Sigma$-protocols for $\Psi_{1}$ and $\Psi_{2}$, there are standard techniques to combine these so as to obtain a $\Sigma$-protocol for $\Psi_{1} \vee \Psi_{2}$ [13]. We define the predicate $\mathrm{DDH}_{S}$, for any server $S$ with triple $S=\left(U_{1}, U_{2}, U_{3}\right)$, as follows:

$$
\mathrm{DDH}_{S}\left(U_{1}, U_{2}, U_{3}\right) \stackrel{\text { def }}{=}\left[\exists x, y \text { s.t. } U_{1}=g_{1}^{x} \wedge U_{2}=g_{1}^{y} \wedge U_{3}=g_{1}^{x y}\right] \text {; }
$$

i.e., $\mathrm{DDH}_{S}$ denotes the predicate asserting that triple $\mathrm{e}_{S}$ is a Diffie-Hellman triple.

The only change in the protocol is the Compute component, which is modified in the following ways (we describe the changes from the point of view of server $A$, but they are applied symmetrically

\footnotetext{
${ }^{7}$ From now on, " $\Sigma$-protocol" means a witness-indistinguishable $\Sigma$-protocol with negligible soundness error.
} 
to server $B$ ): In the first phase, in addition to computing $M_{1} \leftarrow \mathrm{EIG}_{p k_{A}}\left(g_{1}^{-z_{a}}\right)$, server $A$ computes

$$
v_{x_{a}, y_{a}, z_{a}, w_{a}}:=A_{a}^{x_{a}} B_{a}^{y_{a}} C_{a}^{z_{a}} D_{a}^{w_{a}} \quad \text { and } \quad V_{x_{a}, y_{a}, z_{a}, w_{a}} \leftarrow \mathrm{EIG}_{p k_{A}}\left(v_{x_{a}, y_{a}, z_{a}, w_{a}}\right)
$$

and sends $V_{x_{a}, y_{a}, z_{a}, w_{a}}$ to $B$. Define the predicate $\Psi_{1}$ as follows:

$$
\Psi_{1} \stackrel{\text { def }}{=}\left[\begin{array}{rr}
E_{a, 1}=g_{1}^{x_{a}} g_{2}^{y_{a}} h^{z_{a}}\left(c d^{\alpha_{a}}\right)^{w_{a}} \\
\exists x_{a}, y_{a}, z_{a}, w_{a}, r, \tilde{r} \text { s.t. } \quad: \quad \begin{array}{r}
M_{1}=\left(g_{1}^{r}, p k_{A}^{r} \cdot g_{1}^{-z_{a}}\right) \\
\end{array} \quad V_{x_{a}, y_{a}, z_{a}, w_{a}}=\left(g_{1}^{\tilde{r}}, p k_{A}^{\tilde{r}} \cdot A_{a}^{x_{a}} B_{a}^{y_{a}} C_{a}^{z_{a}} D_{a}^{w_{a}}\right)
\end{array}\right] .
$$

Server $A$ then acts as a prover in the protocol $\Sigma\left[\Psi_{1} \vee D \mathrm{H}_{A}\right]$. Meanwhile, $A$ acts as a verifier in the symmetric $\Sigma$-protocol being given (possibly concurrently) by server $B$. If $B$ 's proof fails, then $A$ aborts immediately.

In the second phase of the Compute protocol, in addition to computing $M_{2}$ as in Figure 3, server $A$ also computes

$$
\begin{aligned}
& v_{z_{a}^{\prime}}:=g_{1}^{z_{a}^{\prime}} \quad v_{x_{a}^{\prime}, y_{a}^{\prime}, z_{a}^{\prime}, w_{a}^{\prime}}:=A_{b}^{x_{a}^{\prime}} B_{b}^{y_{a}^{\prime}} C_{b}^{z_{a}^{\prime}} D_{b}^{w_{a}^{\prime}} \\
& V_{z_{a}^{\prime}} \leftarrow \operatorname{EIG}_{p k_{A}}\left(v_{z_{a}^{\prime}}\right) \quad V_{x_{a}^{\prime}, y_{a}^{\prime}, z_{a}^{\prime}, w_{a}^{\prime}} \leftarrow \mathrm{EIG}_{p k_{A}}\left(v_{x_{a}^{\prime}, y_{a}^{\prime}, z_{a}^{\prime}, w_{a}^{\prime}}\right)
\end{aligned}
$$

and sends $V_{z_{a}^{\prime}}$ and $V_{x_{a}^{\prime}, y_{a}^{\prime}, z_{a}^{\prime}, w_{a}^{\prime}}$ to $B$. Define the predicate $\Psi_{2}$ as:

$$
\begin{aligned}
& \Psi_{2} \stackrel{\text { def }}{=}
\end{aligned}
$$

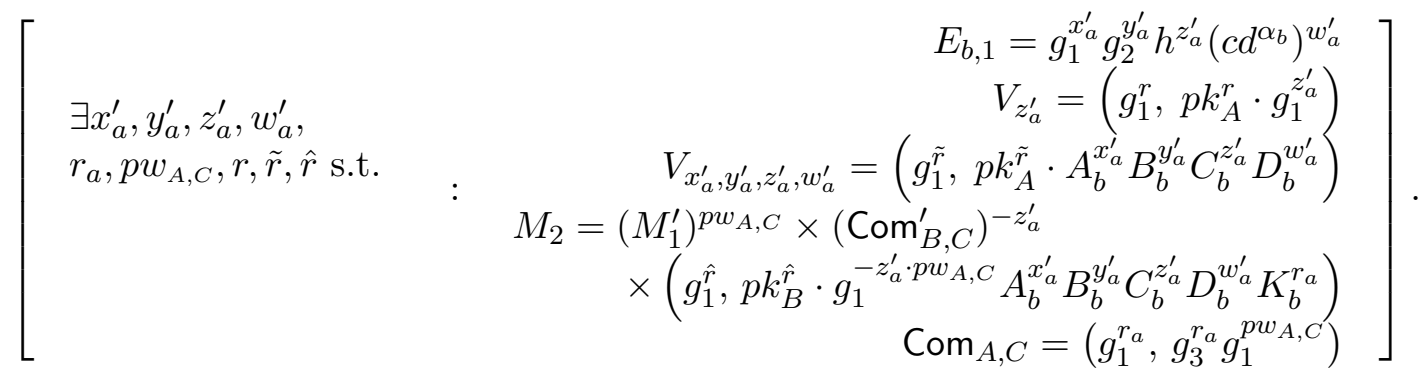

Server $A$ then acts as a prover in the protocol $\Sigma\left[\Psi_{2} \vee \mathrm{DDH}_{A}\right]$. Meanwhile, $A$ acts as a verifier in the symmetric $\Sigma$-protocol being given (possibly concurrently) by server $B$. If $B$ 's proof fails, then $A$ aborts without computing a session key.

Relatively efficient $\Sigma$-protocols for the above predicates can be constructed using standard techniques (see, e.g., [29]), and so we omit further details.

Efficiency. Compared to the basic protocol, the computational work of the client is unchanged. As for the servers, assuming we instantiate the protocol using $\Sigma$-protocols as in [29] we calculate that each server must perform a total of roughly 70 exponentiations. (I.e., each server's work is increased by a factor of roughly 6 as compared to the basic protocol.) Once again, this does not take into account any potential efficiency improvements such as off-line computation or pre-computation that could be done to speed up fixed-base exponentiations. By way of comparison, the protocol of Di Raimondo and Gennaro [16] requires each server to perform roughly 80 exponentiations for the smallest threshold supported by their scheme (i.e., $n=4, t=1$ ). 


\subsection{Proof of Security for Active Adversaries}

We prove that the modified protocol described above is secure against active adversaries; that is:

Theorem 2 With the modifications described above and under the same assumptions as in Theorem 1, we obtain a secure two-server protocol for password-only authenticated key exchange in the presence of an active adversary.

In the proof of the above theorem, we only show the differences from the proof of Theorem 1 . In the following, we let $\mathrm{EIG}_{s k}^{-1}(M) \stackrel{\text { def }}{=} \frac{M[2]}{M[1]^{s k}}$ for any $s k \in \mathbb{Z}_{q}$ and El Gamal ciphertext $M$.

Proof Given an active adversary $\mathcal{A}$ attacking the protocol, we imagine a simulator which runs the protocol for $\mathcal{A}$ as in the proof of Theorem 1. $\operatorname{Adv}_{\mathcal{A}, P}(k)$ is defined as there, and we again refer to the original experiment as $P_{0}$. Throughout the proof, we will again assume without loss of generality that for any client $C$ associated with servers $A$ and $B$, the adversary corrupts server $A$. (The case when neither server is corrupted follows as in the proof for passive adversaries.)

Experiment $P_{0}^{\prime}$ : In experiment $P_{0}^{\prime}$, the simulator makes the following changes: First, as in the proof of Theorem 1, the experiment is aborted and the adversary does not succeed if any of the following occur:

1. At any point, a $\mathrm{msg}_{1}$ generated by the simulator repeats.

2. At any point, a $\mathrm{msg}_{A}$ or $\mathrm{msg}_{B}$ generated by the simulator repeats.

3. At any point, the adversary forges a new, valid message/signature pair for any verification key used in a simulator-generated $\mathrm{msg}_{1}$.

4. At any point during the experiment, a collision occurs in the hash function $H$.

In addition, for any non-corrupted server $B$ the simulator sets triple ${ }_{B}$ to be a (random) DiffieHellman triple, while for any corrupted server $A$ the simulator sets triple ${ }_{A}$ to be a (random) nonDiffie-Hellman triple. Finally, the simulator also changes how it computes the session key for any non-corrupted server $B$; in particular, it will no longer use the long-term El Gamal secret key $s k_{B}$ to compute $X_{b}$. Instead, the simulator computes $X_{b}$ in the following way: using $s k_{A}$, the simulator decrypts $V_{z_{a}^{\prime}}$ and $V_{x_{a}^{\prime}, y_{a}^{\prime}, z_{a}^{\prime}, w_{a}^{\prime}}$ to obtain values $v_{z_{a}^{\prime}}$ and $v_{x_{a}^{\prime}, y_{a}^{\prime}, z_{a}^{\prime}, w_{a}^{\prime}}$, respectively. It then sets:

$$
X_{b}:=g_{1}^{-z_{b} \cdot p w_{C}} \cdot\left(v_{z_{a}^{\prime}}\right)^{-p w_{C}} \cdot K_{b}^{r_{a}} \cdot v_{x_{a}^{\prime}, y_{a}^{\prime}, z_{a}^{\prime}, w_{a}^{\prime}}
$$

(recall that the simulator knows $p w_{C}$ and $r_{a}$ ).

We claim that these changes have only a negligible effect on the adversary's advantage. It is immediate that the four events listed above occur with only negligible probability. Moreover, the DDH assumption implies that the adversary cannot distinguish the change in the way triple ${ }_{S}$ is computed for the corrupted and non-corrupted servers. It remains only to argue that the change in computing $X_{b}$ has negligible effect. Consider an execution of the Compute protocol between a non-corrupted $B$ and a corrupted $A$. Because of the $\Sigma$-protocols executed by $A$ as part of the protocol, with all but negligible probability there exist values $x_{a}^{\prime}, y_{a}^{\prime}, z_{a}^{\prime}, w_{a}^{\prime}$ such that

$$
\mathrm{EIG}_{s k_{B}}^{-1}\left(M_{2}\right)=g_{1}^{-z_{b} \cdot p w_{A, C}} \cdot g_{1}^{-z_{a}^{\prime} \cdot p w_{B, C}} \cdot g_{1}^{-z_{a}^{\prime} \cdot p w_{A, C}} \cdot A_{b}^{x_{a}^{\prime}} B_{b}^{y_{a}^{\prime}} C_{b}^{z_{a}^{\prime}} D_{b}^{w_{a}^{\prime}} K_{a}^{r_{a}}
$$


and

$$
v_{z_{a}^{\prime}}=g_{1}^{z_{a}^{\prime}} \quad \text { and } \quad v_{x_{a}^{\prime}, y_{a}^{\prime}, z_{a}^{\prime}, w_{a}^{\prime}}=A_{b}^{x_{a}^{\prime}} B_{b}^{y_{a}^{\prime}} C_{b}^{z_{a}^{\prime}} D_{b}^{w_{a}^{\prime}},
$$

for values $p w_{A, C}, p w_{B, C}, z_{b}, r_{a}$ chosen (and known) by the simulator. It follows that, with all but negligible probability, the values of $X_{b}$ computed in experiments $P_{0}$ and $P_{0}^{\prime}$ are identical. Putting everything together, we have that $\left|\operatorname{Adv}_{\mathcal{A}, P_{0}}(k)-\operatorname{Adv}_{\mathcal{A}, P_{0}^{\prime}}(k)\right|$ is negligible.

Experiment $P_{0}^{\prime \prime}$ : In this experiment, all $\Sigma$-protocols in which a non-corrupted server $B$ acts as a prover are performed using a witness for the predicate $\mathrm{DDH}_{B}$. (This is possible since, in the previous experiment, triple $_{B}$ was chosen as a DDH triple for any non-corrupted server $B$.) It follows immediately from the witness indistinguishability of the $\Sigma$-protocols that $\left|\operatorname{Adv}_{\mathcal{A}, P_{0}^{\prime}}(k)-\operatorname{Adv}_{\mathcal{A}, P_{0}^{\prime \prime}}(k)\right|$ is negligible.

Experiment $P_{0}^{\prime \prime \prime}$ : Here, all ciphertexts throughout the course of the experiment that are computed by the simulator as El Gamal encryptions of some value with respect to $p k_{B}$ (for a non-corrupted server $B$ ) are now formed by encrypting a random group element. These ciphertexts include the values $\mathrm{Com}_{B, C}^{\prime}$ (for any client $C$ ) constructed during the initialization phase, as well as $M_{1}^{\prime}$ (generated during the Compute protocol run with a corrupted server $A$ ), and all ciphertexts computed by server $B$ as part of the $\Sigma$-protocols in which $B$ acts as a prover when interacting with a corrupted $A$.

Since neither $s k_{B}$ nor the randomness used to generate any of the ciphertexts of the type considered here are ever used by the simulator in experiment $P_{0}^{\prime \prime}$ (relying here on the fact that, in experiment $P_{0}^{\prime \prime}$, the simulator uses a witness for $\mathrm{DDH}_{B}$ when acting as a prover), semantic security of El Gamal encryption implies that $\left|\operatorname{Adv}_{\mathcal{A}, P_{0}^{\prime \prime}}(k)-\operatorname{Adv}_{\mathcal{A}, P_{0}^{\prime \prime \prime}}(k)\right|$ is negligible.

In the experiments that follow, we use the same definitions as in the proof of Theorem 1 for oracle-generated and adversary-generated messages $\mathrm{msg}_{1}$.

Experiment $P_{1}$ : In experiment $P_{1}$, the simulator interacts with the adversary as in $P_{0}^{\prime \prime \prime}$ except for the way the simulator computes certain values in response to a $\mathrm{msg}_{3}$ sent to an uncorrupted server when msg $_{1}$ for the corresponding server instance is oracle generated. (By definition, this is automatically the case for any Execute query being answered by the simulator.) Let $A$ and $B$ be the servers associated with some client $C$, and say $B$ is uncorrupted. Say a $\mathrm{msg}_{3}$ as described above is sent to instance $\Pi_{B}^{j}$, and server $A$ runs the Compute protocol with $B$. The simulator finds the unique $i$ such that $\operatorname{sid}_{C, B}^{i}=\operatorname{sid}_{B}^{j}$ (as in the proof of Theorem 1, a unique such $i$ exists), and sets $\mathrm{sk}_{B, C}^{j}:=\mathrm{sk}_{C, B}^{i}$ (assuming instance $\Pi_{B}^{j}$ does not abort due to an incorrect proof by server $A$ ).

Furthermore, if server $A$ is corrupted then the simulator computes $M_{2}^{\prime}$ in the Compute protocol as follows (again, assuming $\Pi_{B}^{j}$ does not abort due to an incorrect proof by server $A$ ): using $s k_{A}$, the simulator decrypts $M_{1}$ and $V_{x_{a}, y_{a}, z_{a}, w_{a}}$ to obtain $v_{z_{a}} \stackrel{\text { def }}{=} g_{1}^{-z_{a}}$ and $v_{x_{a}, y_{a}, z_{a}, w_{a}}$, respectively. It then sets

$$
M_{2}^{\prime} \leftarrow \mathrm{EIG}_{p k_{A}}\left(\mathrm{sk}_{C, A}^{i} \cdot\left(v_{x_{a}, y_{a}, z_{a}, w_{a}}\right)^{-1} \cdot K_{a}^{-r_{a}} \cdot\left(v_{z_{a}}\right)^{-p w_{A, C}}\right) .
$$

We claim that these changes have only negligible effect on the adversary's advantage. In particular, due to the $\Sigma$-protocols (in which $A$ acts as a prover) we know that with all but negligible probability there exist values $x_{a}^{\prime}, y_{a}^{\prime}, z_{a}^{\prime}, w_{a}^{\prime}$ such that

$$
E_{b, 1}=g_{1}^{x_{a}^{\prime}} g_{2}^{y_{a}^{\prime}} h^{z_{a}^{\prime}}\left(c d^{\alpha_{b}}\right)^{w_{a}^{\prime}} \quad \text { and } \quad v_{z_{a}^{\prime}}=g_{1}^{z_{a}^{\prime}} \quad \text { and } \quad v_{x_{a}^{\prime}, y_{a}^{\prime}, z_{a}^{\prime}, w_{a}^{\prime}}=A_{b}^{x_{a}^{\prime}} B_{b}^{y_{a}^{\prime}} C_{b}^{z_{a}^{\prime}} D_{b}^{w_{a}^{\prime}}
$$


as well as values $x_{a}, y_{a}, z_{a}, w_{a}$ such that

$$
E_{a, 1}=g_{1}^{x_{a}} g_{2}^{y_{a}} h^{z_{a}}\left(c d^{\alpha_{a}}\right)^{w_{a}} \quad \text { and } \quad v_{z_{a}}=g_{1}^{-z_{a}} \quad \text { and } \quad v_{x_{a}, y_{a}, z_{a}, w_{a}}=A_{a}^{x_{a}} B_{a}^{y_{a}} C_{a}^{z_{a}} D_{a}^{w_{a}} .
$$

Assuming this to be the case, consider the computation of $\mathrm{sk}_{B, C}$. In experiment $P_{0}^{\prime \prime \prime}$ we have:

$$
\begin{aligned}
\mathrm{sk}_{B, C}^{j} & =A_{b}^{x_{b}} B_{b}^{y_{b}} C_{b}^{z_{b}} D_{b}^{w_{b}} K_{b}^{r_{b}} \cdot g_{1}^{-z_{b} \cdot p w_{C}} \cdot\left(v_{z_{a}^{\prime}}\right)^{-p w_{C}} \cdot K_{b}^{r_{a}} \cdot v_{x_{a}^{\prime}, y_{a}^{\prime}, z_{a}^{\prime}, w_{a}^{\prime}} \\
& =A_{b}^{x_{b}+x_{a}^{\prime}} B_{b}^{y_{b}+y_{a}^{\prime}}\left(C_{b} / g_{1}^{p w_{C}}\right)^{z_{b}+z_{a}^{\prime}} D_{b}^{w_{b}+w_{a}^{\prime}} K_{b}^{r_{a}+r_{b}} .
\end{aligned}
$$

This is exactly equal to $\mathbf{s k}_{C, B}^{i}$ (for $i$ as defined above), and hence the values of $\mathbf{s k}_{B, C}^{j}$ computed in experiments $P_{0}^{\prime \prime \prime}$ and $P_{1}$ are identical. Considering next the case of $M_{2}^{\prime}$, in experiment $P_{0}^{\prime \prime \prime}$ this is a random El Gamal encryption (with respect to public key $p k_{A}$ ) of:

$$
g_{1}^{-z_{a} \cdot p w_{B, C}} \cdot g_{1}^{-z_{b}^{\prime} \cdot p w_{C}} \cdot A_{a}^{x_{b}^{\prime}} B_{a}^{y_{b}^{\prime}} C_{a}^{z_{b}^{\prime}} D_{a}^{w_{b}^{\prime}} K_{a}^{r_{b}}=\mathrm{sk}_{C, A}^{i} \cdot\left(v_{x_{a}, y_{a}, z_{a}, w_{a}}\right)^{-1} \cdot K_{a}^{-r_{a}} \cdot\left(v_{z_{a}}\right)^{-p w_{A, C}},
$$

for $i$ as defined above. Thus, $M_{2}^{\prime}$ is distributed identically in experiments $P_{0}^{\prime \prime \prime}$ and $P_{1}$. Putting everything together, $\left|\operatorname{Adv}_{\mathcal{A}, P_{1}}(k)-\operatorname{Adv}_{\mathcal{A}, P_{0}^{\prime \prime \prime}}(k)\right|$ is negligible.

Exactly as in the proof of Theorem 1, we next have the simulator choose public parameters $h, c, d$ in such a way that it knows their representations with respect to $g_{1}, g_{2}$. We omit the details here. Once this is done, the simulator can again distinguish between incorrect and correct encryptions, and can thus distinguish between adversary-generated msg $_{1}$ which are valid or invalid; all these terms are defined exactly as in the proof of Theorem 1. We also recall from that proof the notion of an adversary-generated $\mathrm{msg}_{1}$ which re-uses an oracle-generated verification key.

Experiment $P_{2}$ : In this experiment, the simulator changes the way it responds to a $\mathrm{msg}_{3}$ sent to an uncorrupted server when the $\mathrm{msg}_{1}$ for the corresponding server instance is adversary-generated and invalid. Let $A$ and $B$ be the servers associated with some client $C$, and say $B$ is uncorrupted. When msg $_{3}$ as described above is sent to an instance $\Pi_{B}^{j}$ of server $B$ (and this instance does not prematurely terminate the protocol) then $\mathrm{sk}_{B, C}^{j}$ is chosen uniformly at random from $\mathbb{G}$. Furthermore, $M_{2}^{\prime}$ (in the Compute protocol) is computed as an encryption (with respect to public key $p k_{A}$ ) of a uniform element of $\mathbb{G}$.

We prove that $\operatorname{Adv}_{\mathcal{A}, P_{2}}(k)=\operatorname{Adv}_{\mathcal{A}, P_{1}}(k)$ by showing that the distributions on the adversary's views in the two experiments are identical. Let us focus first on the case of $\mathrm{sk}_{B, C}^{j}$. Say

$$
\mathrm{msg}_{1}=\left\langle\text { Client }, \mathrm{VK}, \begin{array}{c}
A_{a}, B_{a}, C_{a}, D_{a} \\
A_{b}, B_{b}, C_{b}, D_{b}
\end{array}\right\rangle
$$

where $\mathrm{msg}_{1}$ is the first message sent to the server instances under consideration. Since $\mathrm{msg}_{1}$ is invalid, we know that (Client, $\mathrm{VK}, A_{b}, B_{b}, C_{b}, D_{b}$ ) is an incorrect encryption. So, it must be the case that either $A_{b}^{\chi_{1}+\alpha_{b} \xi_{1}} B_{b}^{\chi_{2}+\alpha_{b} \xi_{2}} \neq D_{b}$ or else $C_{b} / p w_{\text {Client }} \neq A_{b}^{\kappa}$ (or possibly both). For any $\mu, \nu \in \mathbb{G}$ and fixing the randomness used in the rest of experiment $P_{1}$, the probability over choice of $x_{b}, y_{b}, z_{b}, w_{b}$ that $E_{b, 2}=\mu$ and $\mathbf{s k}_{B, C}^{j}=\nu$ is exactly the probability that

$$
\log \mu=x_{b}+y_{b} \cdot \log g_{2}+z_{b} \cdot \log h+w_{b} \cdot \log \left(c d^{\alpha_{b}}\right)
$$

and

$$
\begin{aligned}
\log \nu-\left(r_{a}+r_{b}\right) \cdot \log K_{b}-\log v_{x_{a}^{\prime}, y_{a}^{\prime}, z_{a}^{\prime}, w_{a}^{\prime}}+p w_{C} \cdot \log v_{z_{a}^{\prime}}= \\
x_{b} \cdot \log A_{b}+y_{b} \cdot \log B_{b}+z_{b} \cdot \log \left(C_{b} / g_{1}^{p w_{C}}\right)+w_{b} \cdot \log D_{b},
\end{aligned}
$$


where all logarithms are with respect to the base $g_{1}$ and $p w_{C}=p w_{\text {Client }}$. As in the proof of Theorem 1, it can be verified that Equations (4) and (5) are linearly independent and not identically zero, when viewed as equations over $\mathbb{Z}_{q}$ in the variables $x_{b}, y_{b}, z_{b}, w_{b}$. Thus, the desired probability is exactly $1 / q^{2}$ and hence the value of $\mathbf{s k}_{B, C}^{j}$ is uniformly distributed in $\mathbb{G}$ independent of the rest

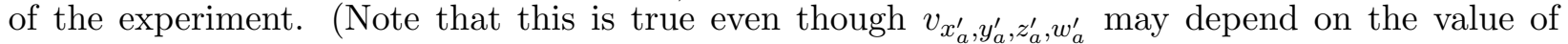
$E_{b, 2}=\mu$, since that is the only dependence of $v_{x_{a}^{\prime}, y_{a}^{\prime}, z_{a}^{\prime}, w_{a}^{\prime}}$ on $x_{b}, y_{b}, z_{b}, w_{b}$.)

A similar argument holds for the values of $M_{2}^{\prime}$ and $E_{a, 2}$, viewed as functions of the random variables $x_{b}^{\prime}, y_{b}^{\prime}, z_{b}^{\prime}, w_{b}^{\prime}$ and using now the fact that (Client, $\mathrm{VK}, A_{a}, B_{a}, C_{a}, D_{a}$ ) is an incorrect en-

cryption. In particular, $\mathrm{Com}_{A, C}^{\prime}$ is an El Gamal encryption (with respect to $p k_{A}$ ) of $g_{1}^{p w_{A, C}}$ and hence the value $M_{2}^{\prime}$ in experiment $P_{1}$ is an El Gamal encryption of the value

$$
\left(\mathrm{EIG}_{s k_{A}}^{-1}\left(M_{1}\right)\right)^{p w_{B, C}} \cdot K_{a}^{r_{b}} \cdot A_{a}^{x_{b}^{\prime}} B_{a}^{y_{b}^{\prime}}\left(C_{a} / g_{1}^{p w_{C}}\right)^{z_{b}^{\prime}} D_{a}^{w_{b}^{\prime}}
$$

The rest of the argument is exactly as above.

The remainder of the proof is essentially the same as the proof of Theorem 1. In fact, the only significant difference is in the counting of the adversary's on-line attacks. Once again the adversary gets the equivalent of two password guesses for each msg ${ }_{1}$ it sends to an uncorrupted server. However, for the adversary to succeed it must either send a valid $\mathrm{msg}_{1}$ to two uncorrupted servers associated with the same client, or else must sent a valid msg $_{1}$ to an uncorrupted server and then initiate the Compute protocol with that same server. Either way, this adversary is charged with two on-line attacks.

\section{Acknowledgments}

The first author would like to thank a very attentive reviewer who helped clarify and correct several aspects of this paper.

\section{References}

[1] M. Bellare, D. Pointcheval, and P. Rogaway. Authenticated Key Exchange Secure Against Dictionary Attacks. Adv. in Cryptology - Eurocrypt 2000, LNCS vol. 1807, Springer-Verlag, pp. 139-155, 2000.

[2] M. Bellare and P. Rogaway. Random Oracles are Practical: A Paradigm for Designing Efficient Protocols. Proc. 1st ACM Conference on Computer and Communications Security, ACM, pp. 62-73, 1993.

[3] M. Bellare and P. Rogaway. Entity Authentication and Key Distribution. Adv. in Cryptology - Crypto 1993, LNCS vol. 773, Springer-Verlag, pp. 232-249, 1994.

[4] M. Bellare and P. Rogaway. Provably Secure Session Key Distribution: the Three Party Case. 27th ACM Symposium on Theory of Computing (STOC), ACM, pp. 57-66, 1995.

[5] S.M. Bellovin and M. Merritt. Encrypted Key Exchange: Password-Based Protocols Secure Against Dictionary Attacks. IEEE Symposium on Research in Security and Privacy, IEEE, pp. 72-84, 1992. 
[6] S.M. Bellovin and M. Merritt. Augmented Encrypted Key Exchange: a Password-Based Protocol Secure Against Dictionary Attacks and Password File Compromise. 1st ACM Conf. on Computer and Comm. Security, ACM, pp. 244-250, 1993.

[7] M. Boyarsky. Public-Key Cryptography and Password Protocols: The Multi-User Case. 7th Ann. Conf. on Computer and Comm. Security, ACM, pp. 63-72, 1999.

[8] V. Boyko, P. MacKenzie, and S. Patel. Provably Secure Password-Authenticated Key Exchange Using Diffie-Hellman. Adv. in Cryptology - Eurocrypt 2000, LNCS vol. 1807, Springer-Verlag, pp. 156-171, 2000.

[9] J. Brainard, A. Juels, B. Kaliski, and M. Szydlo. Nightingale: A New Two-Server Approach for Authentication with Short Secrets. 12th USENIX Security Symp., pp. 201-213, 2003.

[10] R. Canetti, O. Goldreich, and S. Halevi. The Random Oracle Methodology, Revisited. J. ACM 51(4): 557-594, 2004.

[11] R. Canetti, S. Halevi, J. Katz, Y. Lindell, and P. MacKenzie. Universally-Composable Password Authenticated Key Exchange. Adv. in Cryptology - Eurocrypt 2005, LNCS vol. 3494, Springer-Verlag, pp. 404-421, 2005.

[12] R. Cramer. Modular Design of Secure Yet Practical Cryptographic Protocols. PhD Thesis, CWI and University of Amsterdam, 1996.

[13] R. Cramer, I. Damgård, and B. Schoenmakers. Proofs of Partial Knowledge and Simplified Design of Witness Hiding Protocols. Adv. in Cryptology - Crypto 1994, LNCS vol. 839, Springer-Verlag, pp. 174-187, 1994.

[14] R. Cramer and V. Shoup. Design and Analysis of Practical Public-Key Encryption Schemes Secure against Adaptive Chosen Ciphertext Attack. SIAM J. Computing 33(1): 167-226, 2003.

[15] W. Diffie and M. Hellman. New Directions in Cryptography. IEEE Transactions on Information Theory 22(6): 644-654, 1976.

[16] M. Di Raimondo and R. Gennaro. Provably Secure Threshold Password-Authenticated Key Exchange. J. Computer and System Sciences 72(6): 978-1001 (2006).

[17] T. El Gamal. A Public Key Cryptosystem and a Signature Scheme Based on Discrete Logarithms. IEEE Transactions on Information Theory 31: 469-472, 1985.

[18] W. Ford and B.S. Kaliski. Server-Assisted Generation of a Strong Secret from a Password. Proc. 5th IEEE Intl. Workshop on Enterprise Security, 2000.

[19] R. Gennaro and Y. Lindell. A Framework for Password-Based Authenticated Key Exchange. ACM Trans. Information and System Security 9(2): 181-234 (2006).

[20] O. Goldreich and Y. Lindell. Session-Key Generation Using Human Passwords Only. J. Cryptology 19(3): 241-340 (2006). Preliminary version in Crypto 2001.

[21] L. Gong, T.M.A. Lomas, R.M. Needham, and J.H. Saltzer. Protecting Poorly-Chosen Secrets from Guessing Attacks. IEEE J. on Selected Areas in Communications 11(5): 648-656, 1993. 
[22] S. Halevi and H. Krawczyk. Public-Key Cryptography and Password Protocols. ACM Trans. Information and System Security 2(3): 230-268, 1999.

[23] D. Jablon. Strong Password-Only Authenticated Key Exchange. ACM Computer Communications Review 26(5): 5-20, 1996.

[24] D. Jablon. Password Authentication Using Multiple Servers. RSA Cryptographers' Track 2001, LNCS vol. 2020, Springer-Verlag, pp. 344-360, 2001.

[25] S. Jiang and G. Gong. Password Based Key Exchange With Mutual Authentication. Workshop on Selected Areas of Cryptography (SAC), 2004.

[26] J. Katz, R. Ostrovsky, and M. Yung. Efficient Password-Authenticated Key Exchange Using Human-Memorable Passwords. J. ACM 57(1): 78-116, 2009

[27] T.M.A. Lomas, L. Gong, J.H. Saltzer, and R.M. Needham. Reducing Risks from Poorly-Chosen Keys. ACM Operating Systems Review 23(5): 14-18, 1989.

[28] S. Lucks. Open Key Exchange: How to Defeat Dictionary Attacks Without Encrypting Public Keys. Proc. Security Protocols Workshop, LNCS 1361, Springer-Verlag, pp. 79-90, 1997.

[29] P. MacKenzie. An Efficient Two-Party Public-Key Cryptosystem Secure against Adaptive Chosen-Ciphertext Attack. Public Key Cryptography (PKC) 2003, LNCS vol. 2567, SpringerVerlag, pp. 47-61, 2003.

[30] P. MacKenzie, S. Patel, and R. Swaminathan. Password-Authenticated Key Exchange Based on RSA. Intl. J. Information Security 9(6): 387-410, 2010.

[31] P. MacKenzie, T. Shrimpton, and M. Jakobsson. Threshold Password-Authenticated Key Exchange. J. Cryptology 19(1): 27-66 (2006).

[32] V. Shoup. A Proposal for an ISO Standard for Public-Key Encryption, version 2.1. Draft, 2001. Available at http://eprint.iacr.org/2001/112.

[33] M. Szydlo and B. Kaliski. Proofs for Two-Server Password Authentication. RSA Cryptographers' Track 2005, LNCS vol. 3376, Springer-Verlag, pp. 227-244, 2005.

[34] T. Wu. The Secure Remote Password Protocol. Proc. Internet Society Symp. on Network and Distributed System Security, pp. 97-111, 1998. 